TESLA Report 2005-01

\title{
Numerical Studies on the Electro-Optic Sampling of Relativistic Electron Bunches
}

\author{
Sara Casalbuoni ${ }^{1}$, Holger Schlarb ${ }^{1}$, Bernhard Schmidt ${ }^{1}$, Peter Schmüser ${ }^{1,2}$, \\ Bernd Steffen $^{1,2}$, Axel Winter ${ }^{2}$
}

1 Deutsches Elektronen-Synchrotron DESY

2 Institut für Experimentalphysik, Universität Hamburg

\begin{abstract}
At the $1 \mathrm{GeV}$ electron linac of the DESY vacuum-ultraviolet free-electron laser an electro-optic (EO) sampling experiment has been installed permitting to measure the time structure of the bunches with high resolution. The transient electric field of the relativistic bunch corresponds to a sub-picosecond $\mathrm{THz}$ pulse which induces a birefringence in an electro-optic crystal. The sampling of the resulting polarization anisotropy by femtosecond laser pulses is studied in detailed numerical calculations. The $\mathrm{THz}$ and the laser pulses are treated as wave packets which propagate in the zinc telluride resp. gallium phosphide crystals. Using experimental data on the material properties of $\mathrm{ZnTe}$ and $\mathrm{GaP}$ the effects of signal broadening and distortion are explicitely taken into account. The most severe limitation on the time resolution is given by the transverse optical (TO) lattice oscillation in the EO crystal. The lowest TO frequency is $5.3 \mathrm{THz}$ in $\mathrm{ZnTe}$ and $11 \mathrm{THz}$ in GaP. The shortest bunch length which can be resolved with moderate distortion amounts to about $200 \mathrm{fs}$ (FWHM) in ZnTe and 100 fs in GaP. The influence of the crystal thickness on the amplitude and width of the EO signal is studied. The optimum thickness is in the range from 100 to $300 \mu \mathrm{m}$. Multiple internal reflections can be suppressed by using a wedge-shaped EO crystal.
\end{abstract}

\section{Introduction}

The Vacuum Ultraviolet Free-Electron Laser (FEL) at DESY has recently been upgraded to an electron energy of $1 \mathrm{GeV}$, allowing to cover the wavelength range from about 10 to $100 \mathrm{~nm}$. The FEL is based on the principle of Self Amplified Spontaneous Emission (SASE) which opens the way to powerful lasers in the X-ray regime. Electron bunches of extremely high local charge density are needed to achieve laser saturation in the $27 \mathrm{~m}$ long undulator magnet. The $1 \mathrm{nC}$ electron bunches are generated in a radio frequency photocathode, accelerated to relativistic energies and then longitudinally compressed to an rms pulse length of $\sigma_{z}=50 \mu \mathrm{m}$ in a two-stage bunch compression scheme. Precise measurements of the temporal profile of the compressed electron bunches are essential for the optimization of the linac and a proper understanding of the bunch compression mechanism including subtle effects such as coherent synchrotron radiation.

Time measurements with a resolution in the 100 femtosecond regime are at the limit of the best streak cameras available. The electro-optic sampling (EOS) technique $[1,2,3,4,5,6]$ 
has the potential of eventually reaching this resolution. The principle of EOS is as follows: the electric field co-propagating with the relativistic electron bunch induces a birefringence in an optically active crystal like zinc telluride or gallium phosphide, and this optical anisotropy is sampled by an ultrashort polarized laser pulse. Alternatively, the coherent transition radiation pulses produced by the bunches upon crossing a metallized screen can be coupled out of the beam pipe and focused onto the EO crystal.

In this paper we present detailed numerical studies on the electro-optic effect in zinc telluride (ZnTe) and gallium phosphide $(\mathrm{GaP})$. The frequency dependence of the complex index of refraction in the $\mathrm{THz}$ and the optical regime is taken into account and also the frequency dependence of the electro-optic coefficient. The transient electric field of the electron bunch corresponds to an electromagnetic pulse in the $\mathrm{THz}$ regime whose time structure is essentially identical to the time structure of the electron bunch itself if the electrons are highly relativistic (typically for a Lorentz factor $\gamma>1000$ ). Using the available experimental data on the refractive index $n(f)$ and the extinction coefficient $\kappa(f)$ in the $\mathrm{THz}$ regime, we calculate the coupling of this ultrashort $\mathrm{THz}$ pulse into the $\mathrm{EO}$ crystal and its propagation in the material. The frequency dependence of the complex refractive index $n(f)+i \kappa(f)$ leads to a pulse broadening and to distortions of the pulse shape which may become severe for very short bunches. In particular high-frequency oscillations develop in the EO crystal. The propagation and broadening of the laser pulse is also considered. Ideally, both pulses should move with the same speed through the EO crystal to obtain the best time resolution and a maximum signal in the EOS system. The group velocity mismatch constitutes a strong limitation and is investigated in some detail.

The main limitation for the shortest time structure which can be resolved is given by transverse optical (TO) lattice oscillations. The lowest TO frequency amounts to 5.3 $\mathrm{THz}$ for $\mathrm{ZnTe}$ and $11 \mathrm{THz}$ for GaP. Near a resonance the refractive index is rapidly changing and it is basically impossible to achieve equal group velocities of the $\mathrm{THz}$ and the laser pulse. Obviously, GaP is better suited to measure very short pulses owing to its higher TO frequency. The disadvantage is that the electro-optic coefficient of GaP is a factor of eight lower than that of ZnTe.

The paper is organized as follows. The theoretical background of the EO effect is presented in Sect. 2 which has been adapted from a previous report [7]. In Sect. 3 we collect the available experimental data on the relevant material properties of ZnTe and GaP. The principles of the mathematical and numerical treatment are explained in Sect 4. The studies on the reconstruction of electron bunches of various length and shape are presented in Sect. 5. The influence of material parameter variations is studied in Appendix A. The comparison between the conventional approach to compute the EO signal by means of an electro-optic response function and the pulse propagation method is presented in Appendix B. 


\section{Electro-Optic Effects in Anisotropic Crystals}

For a homogeneous medium the electric displacement vector is given by

$$
\boldsymbol{D}=\varepsilon_{0} \varepsilon \boldsymbol{E}
$$

where the (relative) dielectric permittivity $\varepsilon$ is a scalar quantity (independent of direction). In a crystal the polarization may depend on the direction of the electric field with respect to the crystallographic axes. In this case the permittivity is a symmetric tensor $\widehat{\varepsilon}$ and $\boldsymbol{D}$ is in general not parallel to the electric field. It is always possible to carry out a principalaxis transformation to an orthogonal coordinate system in which $\boldsymbol{E}$ and $\boldsymbol{D}$ are related by a diagonal matrix

$$
\left(\begin{array}{c}
D_{1} \\
D_{2} \\
D_{3}
\end{array}\right)=\varepsilon_{0}\left(\begin{array}{ccc}
\varepsilon_{1} & 0 & 0 \\
0 & \varepsilon_{2} & 0 \\
0 & 0 & \varepsilon_{3}
\end{array}\right) \cdot\left(\begin{array}{c}
E_{1} \\
E_{2} \\
E_{3}
\end{array}\right)
$$

If the $\varepsilon_{i}$ are not all identical the crystal exhibits birefringence (double refraction).

The energy density of the electric field is $w_{e}=\frac{1}{2} \boldsymbol{E} \cdot \boldsymbol{D}$. Using Eq. (2) we can show that the surfaces of constant energy density are ellipsoids in the $\boldsymbol{D}$ space:

$$
\varepsilon_{0} w_{e}=\boldsymbol{D} \cdot \widehat{\varepsilon}^{-1} \cdot \boldsymbol{D}=\frac{D_{1}^{2}}{\varepsilon_{1}}+\frac{D_{2}^{2}}{\varepsilon_{2}}+\frac{D_{3}^{2}}{\varepsilon_{3}} .
$$

Defining a dimensionless vector along the direction of $\boldsymbol{D}$ by $\boldsymbol{u}=\boldsymbol{D} / \sqrt{2 \varepsilon_{0} w_{e}}$ we get the equation of the refractive index ellipsoid

$$
\frac{u_{1}^{2}}{n_{1}^{2}}+\frac{u_{2}^{2}}{n_{2}^{2}}+\frac{u_{3}^{2}}{n_{3}^{2}}=1
$$

where as usual $n_{i}=\sqrt{\varepsilon_{i}}$ for a nonmagnetic material. Defining the impermeability tensor by

$$
\widehat{\boldsymbol{\eta}}=\widehat{\varepsilon}^{-1}
$$

the ellipsoid equation can be written as

$$
\boldsymbol{u} \cdot \widehat{\boldsymbol{\eta}} \cdot \boldsymbol{u}=1
$$

\subsection{Electro-optic effect in zinc telluride and gallium phosphide}

ZnTe and GaP have a cubic crystal lattice and are optically isotropic at vanishing electric field which means that the impermeability tensor can be replaced by the scalar quantity $\varepsilon^{-1}$, multiplied with the unit matrix $\boldsymbol{I}$. In the presence of an electric field the impermeability tensor becomes

$$
\widehat{\boldsymbol{\eta}}(\boldsymbol{E})=\varepsilon^{-1} \boldsymbol{I}+\boldsymbol{r} \cdot \boldsymbol{E} .
$$

The second term describes the Pockels effect. The Kerr effect which is quadratic in the electric field is neglected here. The ellipsoid equation is now

$$
\boldsymbol{u} \cdot \widehat{\boldsymbol{\eta}}(\boldsymbol{E}) \cdot \boldsymbol{u}=\sum_{i, j=1,2,3}\left(\varepsilon^{-1} \delta_{i j}+\sum_{k=1,2,3} r_{i j k} E_{k}\right) u_{i} u_{j}=1
$$


The tensor $\widehat{\boldsymbol{\eta}}$ is symmetric, hence $r_{i j k}=r_{j i k}$. It is convention to replace the first two indices $i, j$ of the tensor $\boldsymbol{r}$ by a single index:

$$
\begin{array}{|c|c|}
\hline(1,1) \rightarrow 1 & r_{11 k} \rightarrow r_{1 k} \\
(2,2) \rightarrow 2 & r_{22 k} \rightarrow r_{2 k} \\
(3,3) \rightarrow 3 & r_{33 k} \rightarrow r_{3 k} \\
(2,3) \rightarrow 4 & r_{23 k}=r_{32 k} \rightarrow r_{4 k} \\
(1,3) \rightarrow 5 & r_{13 k}=r_{31 k} \rightarrow r_{5 k} \\
(1,2) \rightarrow 6 & r_{12 k}=r_{21 k} \rightarrow r_{6 k} \\
\hline
\end{array}
$$

ZnTe and GaP crystallize in the zincblende structure (two face-centered cubic lattices which are shifted against each other by one quarter of the spatial diagonal). Owing to the high degree of symmetry, the $\mathrm{ZnTe}(\mathrm{GaP})$ crystal is optically isotropic at vanishing electric fields, i.e. $n_{1}=n_{2}=n_{3}=n_{0}$. In addition, the tensor $\boldsymbol{r}$ contains only one independent element: $r_{41}=r_{52}=r_{63}$. Hence the equation of the refractive index ellipsoid assumes the form

$$
\frac{1}{n_{0}^{2}}\left(u_{1}^{2}+u_{2}^{2}+u_{3}^{2}\right)+2 r_{41}\left(E_{1} u_{2} u_{3}+E_{2} u_{3} u_{1}+E_{3} u_{1} u_{2}\right)=1 .
$$

To obtain the modified refractive indices one has to perform a principal-axis transformation.

\subsection{Determination of the main refractive indices}

The ZnTe (GaP) crystals used in EOS experiments are cut in the (110) plane as shown in Fig. 1. The THz pulse and the laser pulse impinge perpendicular to this plane along the direction $[-1,-1,0]$, their electric vectors lie therefore in the (110) plane. We define a two-dimensional coordinate system $(X, Y)$ in this plane where $X$ points along the $[-1,1,0]$ direction and $Y$ along the $[0,0,1]$ direction.
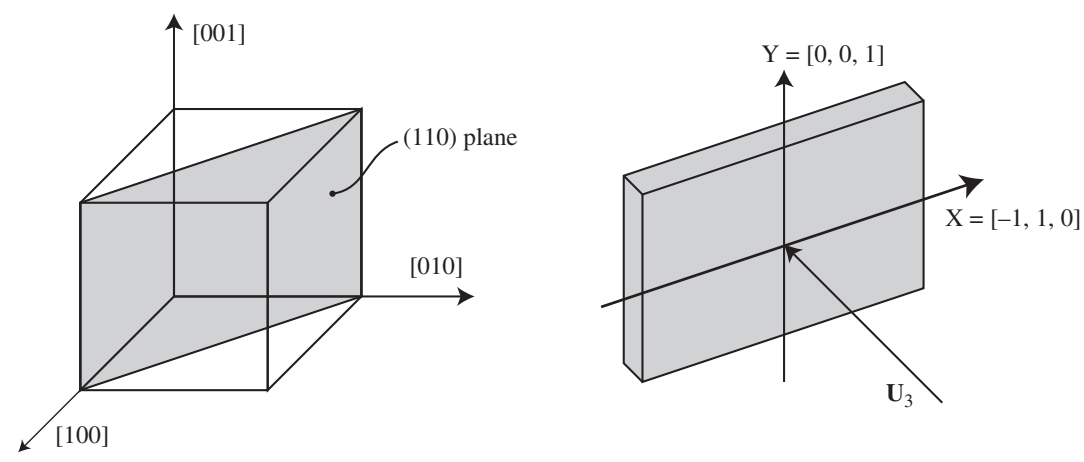

Figure 1: Left: The (110) plane in the cubic zinc telluride crystal. Right: The coordinate system $(X, Y)$ in the (110) plane. The $\mathrm{THz}$ and laser pulses impinge along the direction $\boldsymbol{U}_{3}$ which is the normal to this plane.

Let the electric vector $\boldsymbol{E}_{a}$ of the THz pulse enclose an angle $\alpha$ with the $X$ axis (the $[-1,1,0]$ axis of the ZnTe resp. GaP crystal). Its components in the base system of the 
cubic crystal lattice are

$$
\boldsymbol{E}_{a}=E_{a}\left(\begin{array}{c}
-\cos \alpha / \sqrt{2} \\
\cos \alpha / \sqrt{2} \\
\sin \alpha
\end{array}\right)
$$

Equation (9) reads in this special case

$$
\boldsymbol{u} \cdot \widehat{\boldsymbol{\eta}}\left(\boldsymbol{E}_{a}\right) \cdot \boldsymbol{u}=1
$$

with the field-dependent impermeability tensor

$$
\widehat{\boldsymbol{\eta}}\left(\boldsymbol{E}_{a}\right)=\frac{1}{n_{0}^{2}}\left(\begin{array}{ccc}
1 & 0 & 0 \\
0 & 1 & 0 \\
0 & 0 & 1
\end{array}\right)+r_{41} E_{a}\left(\begin{array}{ccc}
0 & \sin \alpha & \cos \alpha / \sqrt{2} \\
\sin \alpha & 0 & -\cos \alpha / \sqrt{2} \\
\cos \alpha / \sqrt{2} & -\cos \alpha / \sqrt{2} & 0
\end{array}\right)
$$

The eigenvalues of the tensor are

$$
\lambda_{1,2}=\frac{1}{n_{0}^{2}}-\frac{r_{41} E_{a}}{2}\left(\sin \alpha \pm \sqrt{1+3 \cos ^{2} \alpha}\right), \quad \lambda_{3}=\frac{1}{n_{0}^{2}}+r_{41} E_{a} \sin \alpha,
$$

and the normalized eigenvectors

$$
\begin{aligned}
& \boldsymbol{U}_{1}=\frac{1}{2} \sqrt{1+\frac{\sin \alpha}{\sqrt{1+3 \cos ^{2} \alpha}}}\left(\begin{array}{c}
-1 \\
1 \\
\frac{2 \sqrt{2} \cos \alpha}{\sqrt{1+3 \cos ^{2} \alpha+\sin \alpha}}
\end{array}\right) \\
& \boldsymbol{U}_{2}=\frac{1}{2} \sqrt{1-\frac{\sin \alpha}{\sqrt{1+3 \cos ^{2} \alpha}}}\left(\begin{array}{c}
1 \\
-1 \\
\frac{2 \sqrt{2} \cos \alpha}{\sqrt{1+3 \cos ^{2} \alpha}-\sin \alpha}
\end{array}\right) \\
& \boldsymbol{U}_{3}=\frac{1}{\sqrt{2}}\left(\begin{array}{c}
-1 \\
-1 \\
0
\end{array}\right) .
\end{aligned}
$$

The principal axes point in the direction of the eigenvectors. The main refractive indices are given by

$$
n_{i}=1 / \sqrt{\lambda_{i}}
$$

Considering that $r_{41} E_{a} \ll 1 / n_{0}^{2}$ this yields in good approximation

$$
\begin{aligned}
& n_{1}=n_{0}+\frac{n_{0}^{3} r_{41} E_{a}}{4}\left(\sin \alpha+\sqrt{1+3 \cos ^{2} \alpha}\right) \\
& n_{2}=n_{0}+\frac{n_{0}^{3} r_{41} E_{a}}{4}\left(\sin \alpha-\sqrt{1+3 \cos ^{2} \alpha}\right) \\
& n_{3}=n_{0}-\frac{n_{0}^{3} r_{41} E_{a}}{2} \sin \alpha .
\end{aligned}
$$

From Eq. (13) it is obvious that the third principal axis is perpendicular to the (110) crystal plane $^{1}$. This is also the direction of incidence of the THz pulse and the Ti:Sa Laser

\footnotetext{
${ }^{1}$ The normal to this plane is the unit vector $\boldsymbol{U}_{3}$.
} 
pulse. The vector $\boldsymbol{U}_{1}$ lies in the (110) plane and encloses an angle $\psi$ with the $[-1,1,0]$ axis which can be calculated using the scalar product. Using the relation $\cos (2 \psi)=2 \cos ^{2} \psi-1$ one gets

$$
\cos 2 \psi=\frac{\sin \alpha}{\sqrt{1+3 \cos ^{2} \alpha}} .
$$

The second principal axis is parallel to the vector $\boldsymbol{U}_{2}$ and of course perpendicular to $\boldsymbol{U}_{1}$. Note that some care is needed to evaluate the vector $\boldsymbol{U}_{2}$ in the limit $\alpha \rightarrow \pi / 2$ since the normalization factor vanishes here while the third component tends to infinity. One finds

$$
\boldsymbol{U}_{2}\left(\frac{\pi}{2}\right)=\left(\begin{array}{l}
0 \\
0 \\
1
\end{array}\right)
$$

So for $\alpha=\pi / 2$ the principal axis $\boldsymbol{U}_{1}$ points in the $X$ direction, and the axis $\boldsymbol{U}_{2}$ in the $Y$ direction.

The principal refractive indices corresponding to the first two principal axes are $n_{1}$ and $n_{2}$. The refractive index ellipse is shown in Fig. 2.

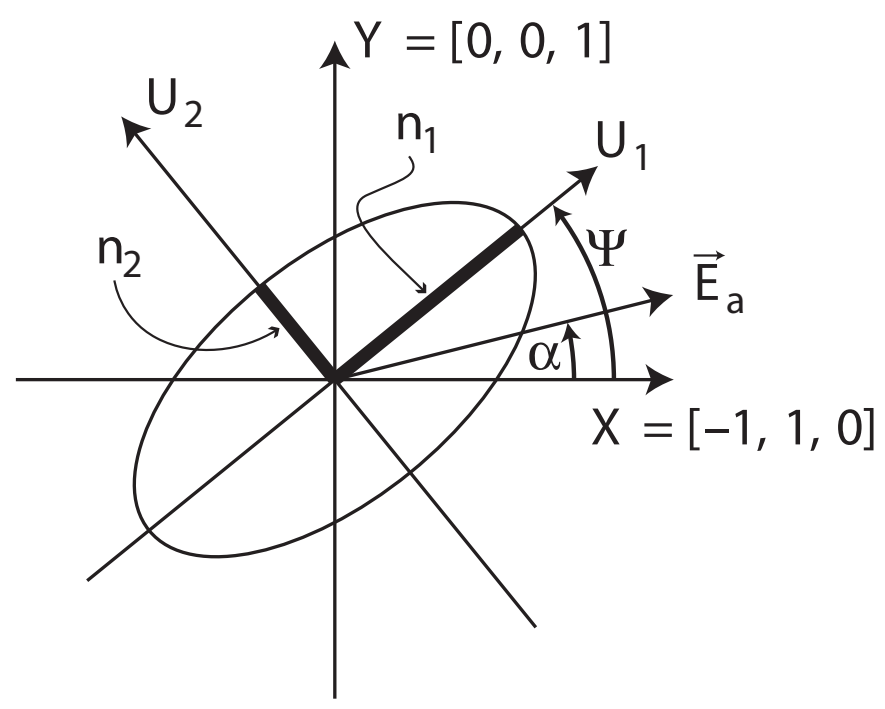

Figure 2: The refractive index ellipsoid projected onto the (110) plane of the zinc telluride crystal. The difference between the refractive indices $n_{1}$ and $n_{2}$ is strongly exaggerated. The electric vector $\boldsymbol{E}_{a}$ encloses an angle $\alpha$ with the $X=[-1,1,0]$ axis of the ZnTe crystal while the angle between the long half axis of the ellipse and the $X$ axis is given by $\psi(\alpha)$. Both the $\mathrm{THz}$ and laser pulses impinge along the normal to the (110) plane, given by the unit vector $\boldsymbol{U}_{3}=(-1 / \sqrt{2},-1 / \sqrt{2}, 0)$.

The Ti:Sa laser beam is incident on the ZnTe $(\mathrm{GaP})$ crystal along the direction $\boldsymbol{U}_{3}=$ $[-1 / \sqrt{2},-1 / \sqrt{2}, 0]$. Its electric vector $\boldsymbol{E}_{b}$ lies therefore in the (110) plane. In the crystal of thickness $d$ the two components of $\boldsymbol{E}_{b}$ along the principal axes $\boldsymbol{U}_{1}$ and $\boldsymbol{U}_{2}$ receive a relative phase shift

$$
\Gamma=\frac{\omega_{0} d}{c}\left(n_{1}-n_{2}\right)=\frac{\pi d}{\lambda_{0}} n_{0}^{3} r_{41} E_{a} \sqrt{1+3 \cos ^{2} \alpha},
$$



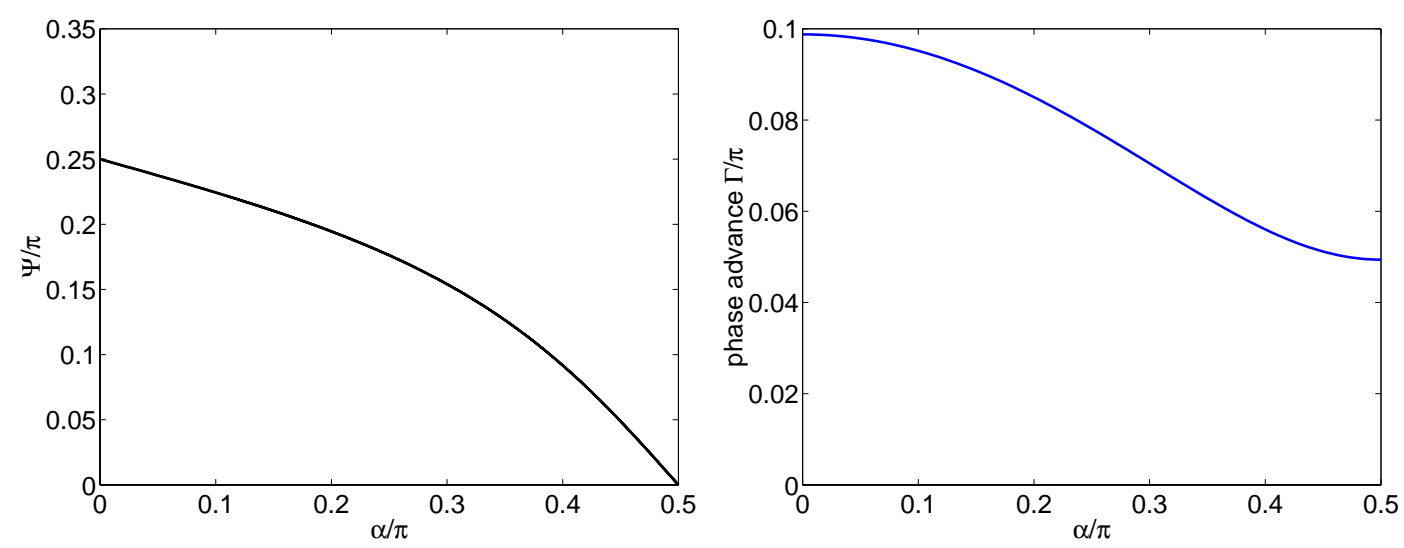

Figure 3: Left: The angle $\psi$ between the first principal axis of the refractive index ellipsoid and the $X$ axis as a function of the angle $\alpha$ between the electric vector $\boldsymbol{E}_{a}$ of the $\mathrm{THz}$ field and the $X$ axis. Right: The retardation parameter $\Gamma$, i.e. the relative phase shift between the two orthogonal components of the laser field $\boldsymbol{E}_{b}$, plotted as a function of $\alpha$. The curve is computed for a ZnTe crystal of $d=500 \mu \mathrm{m}$ thickness and a $\mathrm{THz}$ field $E_{a}=10^{6} \mathrm{~V} / \mathrm{m}$.

where $\omega_{0}$ is the angular frequency of the laser light and $\lambda_{0}$ its wavelength in vacuum. The rotation angle $\psi$ of the index ellipse in the $(X, Y)$ plane and the relative phase shift $\Gamma$, called retardation parameter in the following, are plotted in Fig. 3 as a function of the angle $\alpha$ between the electric vector $\boldsymbol{E}_{a}$ of the THz field and the $X$ axis.

\subsection{Principle of signal detection}

Here we give only a short summary of the signal detection scheme, for a detailed treatment we refer to [7]. A high sensitivity can be achieved with a balanced diode detector. For that purpose the laser beam leaving the ZnTe (GaP) crystal passes through a quarter wave plate whose main axes are oriented at $\pm 45^{\circ}$ with respect to the horizontal direction (i.e. the $X$ axis). A Wollaston prism separates the two polarization components and guides them to the two diodes of a balanced detector. The arrangement is sketched in Fig. 4.

If the electric field of the bunch (Terahertz field) is absent the Ti:Sa laser pulse remains unaffected by the ZnTe resp. GaP crystal and leaves it with horizontal polarization. The quarter wave plate transforms this to circular polarization. The Wollaston prism guides the two orthogonal components of the circular wave to the two diodes which record then of course the same intensity. Hence the difference signal vanishes. In the presence of a Terahertz field, however, the radiation behind the ZnTe (GaP) crystal is elliptically polarized leading to an imbalance between the two diode signals. The difference signal is proportional to the sine of the retardation parameter [7]

$$
\left|A_{1}\right|^{2}-\left|A_{2}\right|^{2} \propto \sin (\Gamma)
$$

where $A_{1}$ and $A_{2}$ are the light amplitudes in detector 1 resp. detector 2 . 


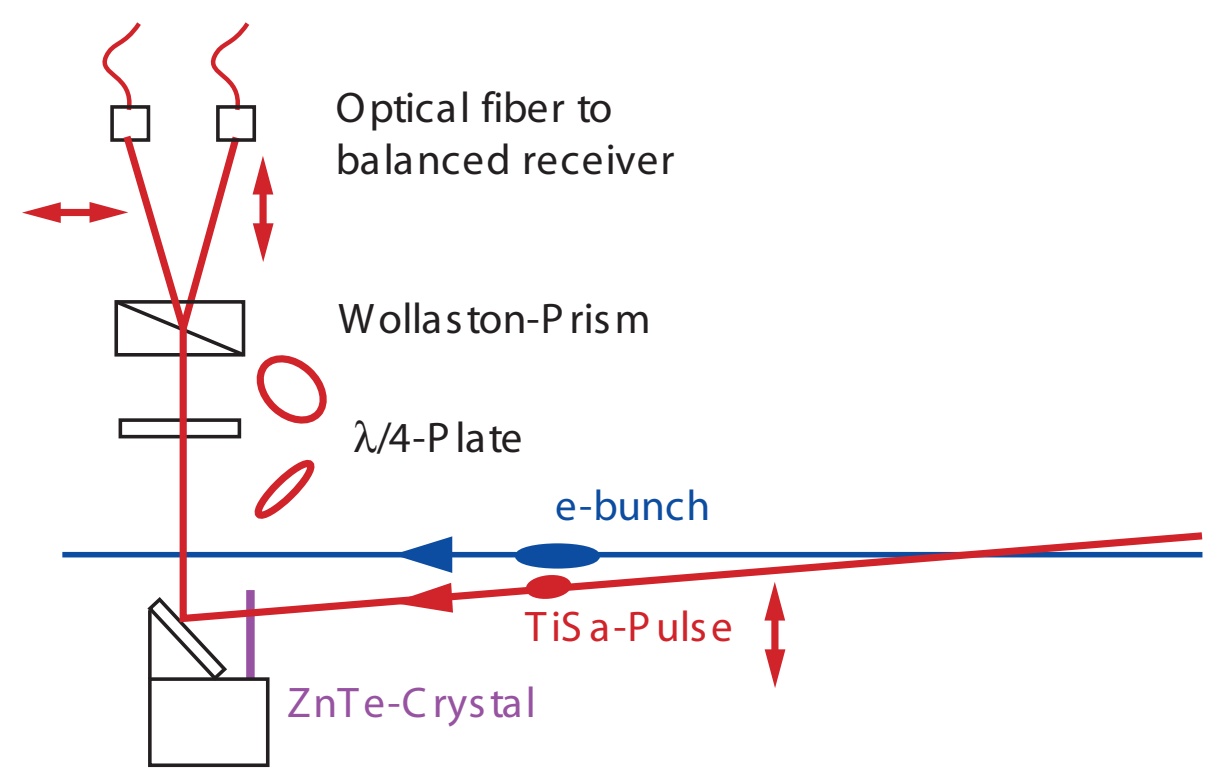

Figure 4: Simplified view of electro-optic signal detection using a quarter wave plate, a Wollaston prism and a balanced diode detector. The laser is polarized horizontally. The quarter wave plate is rotated by $45^{\circ}$ with respect to the horizontal plane.

\section{Electro-Optic Properties of ZnTe and GaP}

\subsection{Refractive index}

\subsubsection{Visible and infrared light}

The index of refraction for visible and infrared light has been measured both for ZnTe $[8,9]$ and GaP [10]. A useful parametrization for ZnTe is [8]

$$
n(\lambda)=\sqrt{4.27+\frac{3.01 \lambda^{2}}{\lambda^{2}-0.142}}
$$

where $\lambda$ is the photon wavelength in $\mu \mathrm{m}$. The optical refractive index of GaP is described by a similar expression ${ }^{2}$

$$
n(\lambda)=\sqrt{2.680+\frac{6.40 \lambda^{2}}{\lambda^{2}-0.0903279}} .
$$

The refractive indices of ZnTe and GaP are respectively plotted in Fig. 5 and Fig. 6 as a function of the wavelength in $\mu \mathrm{m}$.

\subsubsection{THz range}

For frequencies far below the optical regime the complex dielectric function $\varepsilon(f)$ of a non-conducting crystal can be written in the form [13]

$$
\varepsilon(f)=\varepsilon_{e l}+\sum_{j} \frac{S_{j} f_{j}^{2}}{f_{j}^{2}-f^{2}-i \Gamma_{j} f}
$$

\footnotetext{
${ }^{2} \mathrm{~A}$ parametrization in terms of the photon energy has been published by Pikhtin et al. [10].
} 


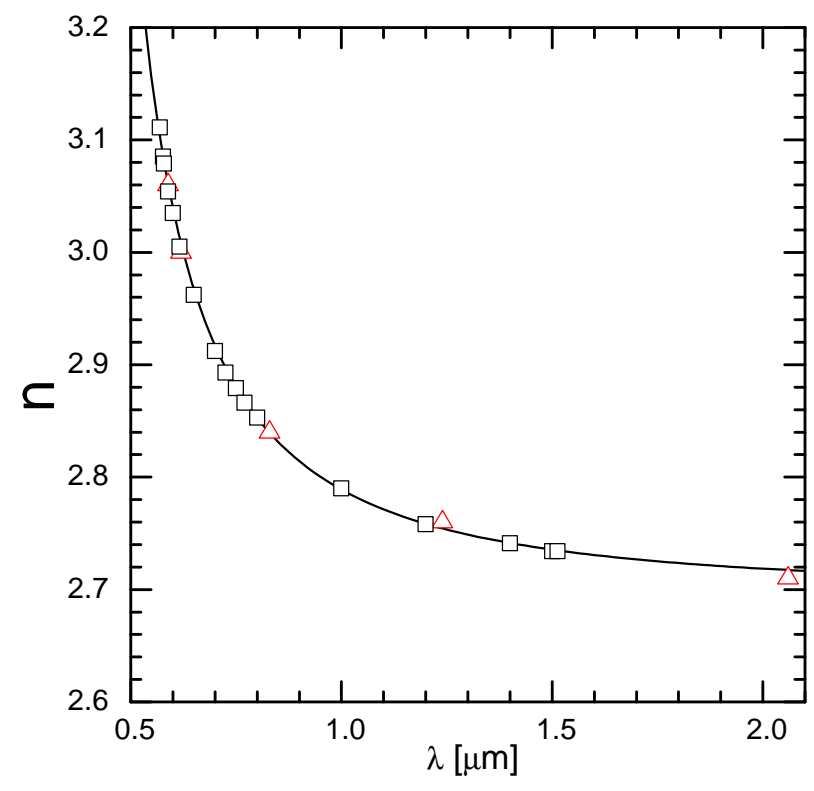

Figure 5: The refractive index of ZnTe for visible and infrared light. Squares: experimental data [9], triangles: experimental data [8], solid curve: fit according to Eq. (18).

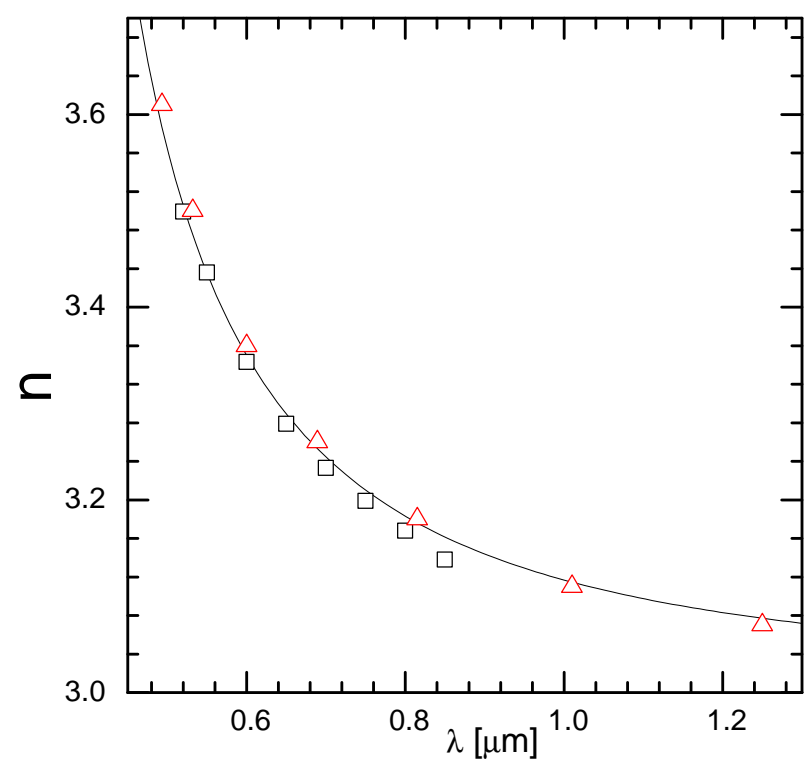

Figure 6: Refractive index of GaP. The experimental data are indicated by squares [11] resp. triangles [12], solid curve: fit according to Eq. (19). 
where the first term is the contribution from the bound electrons and the second term the contribution from lattice oscillations which are treated as damped harmonic oscillators. The coefficient $\varepsilon_{e l}$ is constant in the $\mathrm{THz}$ frequency range. The sum extends over all lattice oscillations which couple to the electromagnetic field. The quantities $f_{j}, \Gamma_{j}$ and $S_{j}$ are the eigenfrequency, damping constant and oscillator strength of the lattice oscillation $j$. For the electro-optic crystals ZnTe and GaP, a good description of $\varepsilon(f)$ in the $\mathrm{THz}$ regime is obtained by restricting the sum to the lowest transverse-optical (TO) lattice oscillation:

$$
\varepsilon(f)=\varepsilon_{e l}+\frac{S_{0} f_{0}^{2}}{f_{0}^{2}-f^{2}-i \Gamma_{0} f} .
$$

The complex index of refraction is given by taking the square root:

$$
n(f)+i \kappa(f)=\sqrt{\varepsilon(f)}
$$

In Fig. 7 and in Fig. 8 we plot the published experimental data for ZnTe resp. GaP on the real and imaginary part of the refractive index as a function of frequency. For ZnTe we observe a variation up to $25 \%$ between the data from Ref. [14] and those from Ref. [9]. Thus, we have used two different sets of fitting parameters. For Ref. [14] we use:

$$
\text { ZnTe : } \varepsilon_{e l}=7.4, \quad f_{0}=5.3 \mathrm{THz}, \quad S_{0}=2.7, \quad \Gamma_{0}=0.09 \mathrm{THz}
$$

while for Ref. [9]:

$$
\text { ZnTe : } \varepsilon_{e l}=6.5, \quad f_{0}=5.35 \mathrm{THz} . \quad S_{0}=2.0, \quad \Gamma_{0}=0.09 \mathrm{THz} .
$$

For the simulations in the following sections we consider the first set of parameters. We will discuss the second set of parameters in appendix A where we analyze the influence of parameter uncertainties on the simulated EOS signal.

The corresponding parameters used for $\mathrm{GaP}$ are:

$$
\mathrm{GaP}: \quad \varepsilon_{e l}=8.7, \quad f_{0}=10.98 \mathrm{THz}, \quad S_{0}=1.8, \quad \Gamma_{0}=0.02 \mathrm{THz}
$$



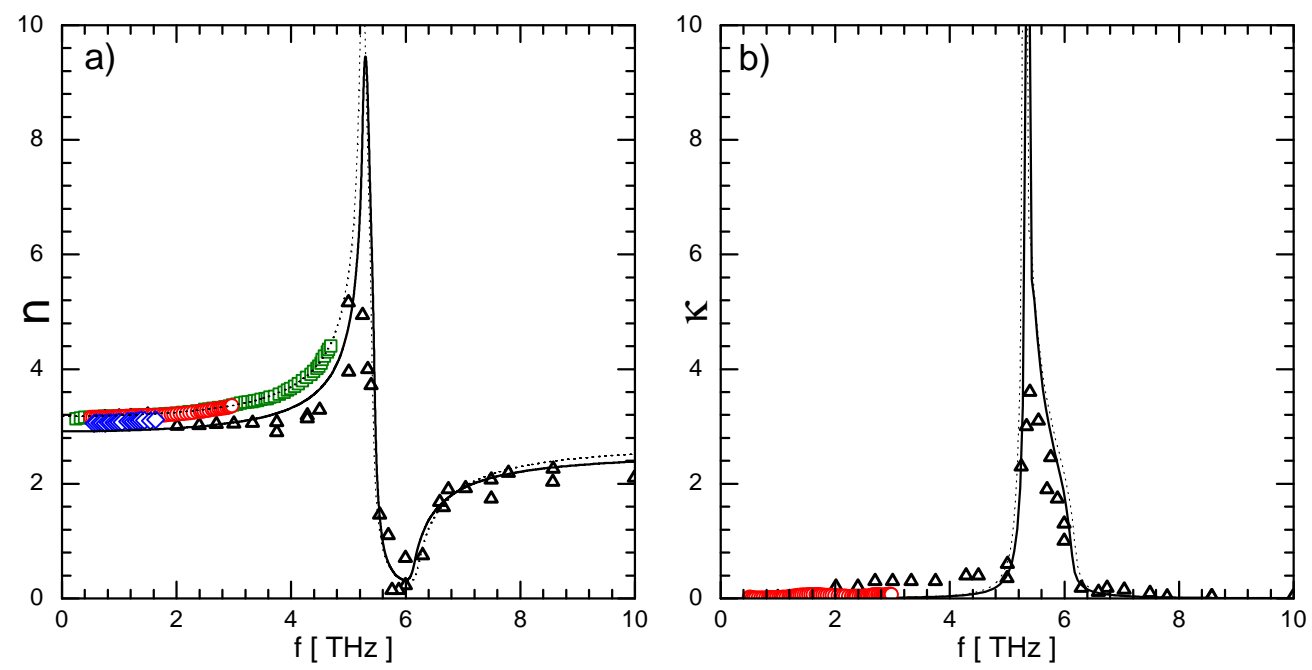

Figure 7: a) Real part $n(f)$ and b) imaginary part $\kappa(f)$ of the refractive index of ZnTe in the $\mathrm{THz}$ range. The resonance at $5.3 \mathrm{THz}$ is due to the excitation of a transverse optical (TO) lattice oscillation. The square symbols $\square$ are experimental data taken from Ref. [14], the triangles $\triangle$ from Ref. [9], the diamonds $\diamond$ from Ref. [15] and the circles $\circ$ from Ref. [16]. The curves are fits of the experimental data using the formulas and the parameters shown in the text.
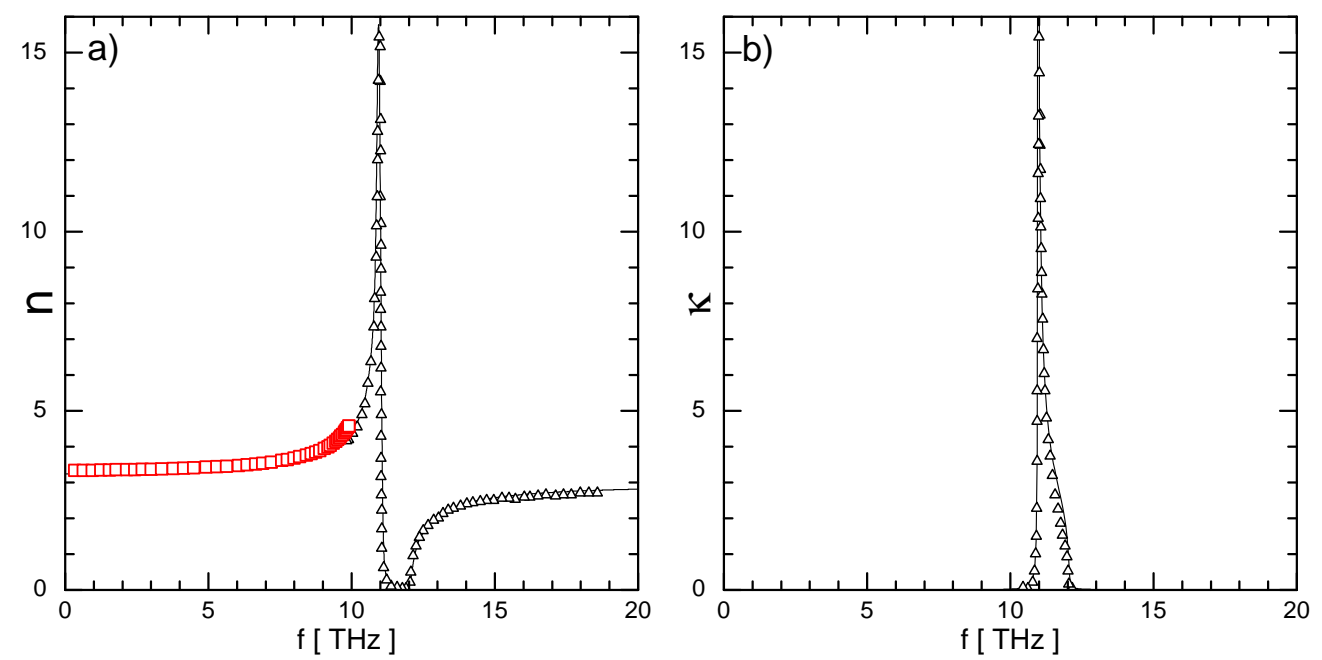

Figure 8: a) $n(f)$ and b) $\kappa(f)$ for GaP. Here the lowest TO resonance is at $11 \mathrm{THz}$. The triangles $\triangle$ are experimental data taken from Ref. [17], the squares $\square$ from Ref. [18]. The curves are fits of the experimental data using the formulas and the parameters shown in the text. 


\subsection{Electro-optic coefficient}

The electro-optic coefficient $r_{41}$ is also influenced by lattice oscillations. It can be written as a function of frequency in the form [19]

$$
r_{41}(f)=d_{E}\left(1+\frac{C f_{0}^{2}}{f_{0}^{2}-f^{2}-i \Gamma_{0} f}\right)
$$

with the following parameters for ZnTe:

$$
\text { ZnTe : } d_{E}=4.25 \cdot 10^{-12} \mathrm{~m} / \mathrm{V}, C=-0.07, f_{0}=5.3 \mathrm{THz}, \Gamma_{0}=0.09 \mathrm{THz} \text {. }
$$

The value of $C$ has been taken from Ref. [20]. In Fig. 9 we show published data on $r_{41}$ as a function of frequency for ZnTe. The curve shows the frequency dependence given by Eq. (23). The very small value of $r_{41}$ at $30 \mathrm{THz}$, reported in Ref. [21], is in gross disagreement with other data [22] and the values found in the visible range [23]. A possible reason for this discrepancy might be the low resistivity value of $\rho \approx 10^{3} \Omega \mathrm{m}$ of the sample of Ref. [21] while $\rho \approx 4 \times 10^{6} \Omega \mathrm{m}$ in Ref. [23] and of $\rho \approx 10^{7} \Omega \mathrm{m}$ in Ref. [22]. For this reason we disregard the data point from Ref. [21]. The experimental data are too scarce and imprecise to derive a frequency dependence of $r_{41}$. On the other hand, due to the low piezoelectric constant of ZnTe [24, 23], the value of the constant $C$ is expected to be small, so that the high frequency value $r_{41}(\infty)$ should not differ appreciably from the low frequency value $r_{41}(0)$. For our simulations we therefore assume a constant value of $r_{41}(f)=4.0 \cdot 10^{-12} \mathrm{~m} / \mathrm{V}$. We have verified that no significant change in the simulated EO signal is observed if instead of a constant $r_{41}$ the frequency dependence given by Eq. (23) is used.

The literature values on $r_{41}$ for GaP are collected in Fig. 10. In the following sections we use for GaP the frequency dependence given in Eq. (23) with the following parameters:

$$
\mathrm{GaP}: d_{E}=1 \cdot 10^{-12} \mathrm{~m} / \mathrm{V}, C=-0.53, f_{0}=10.98 \mathrm{THz}, \Gamma_{0}=0.02 \mathrm{THz} \text {. }
$$




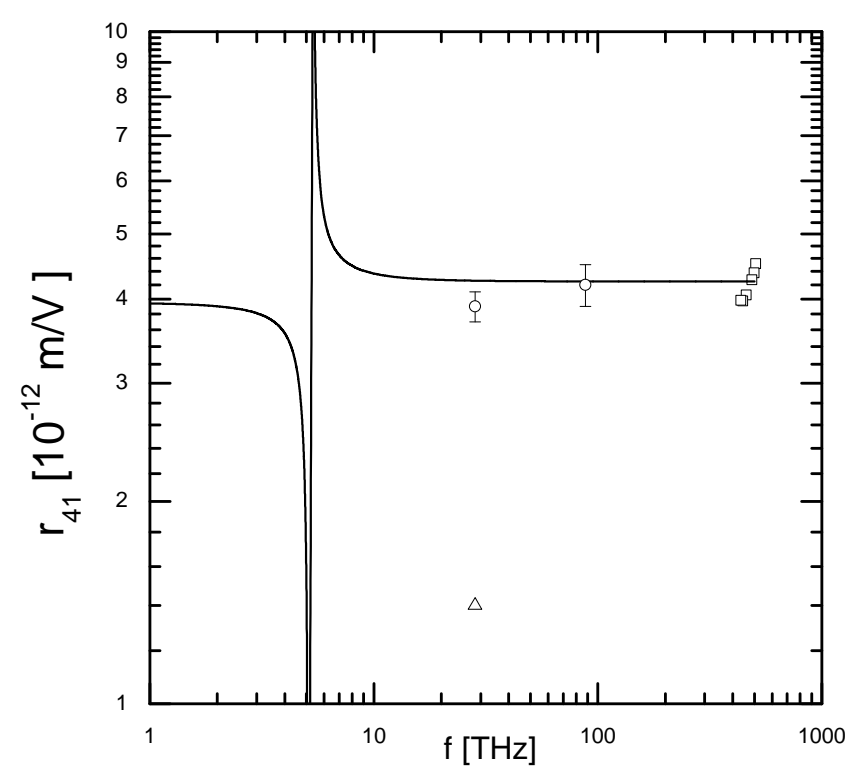

Figure 9: The EO coefficient $r_{41}(f)$ of $Z n T e$. The square symbols $\square$ are experimental data taken from Ref. [23] $\left(\rho \approx 4 \times 10^{6} \Omega \mathrm{m}\right)$, the circles $\circ$ from Ref. [22] $\left(\rho \approx 10^{7} \Omega \mathrm{m}\right)$, the triangle $\triangle$ from Ref. [21] ( $\rho \approx 10^{3} \Omega \mathrm{m}$ ). The curve is obtained using Eq. (23) with the parameters shown in the text.

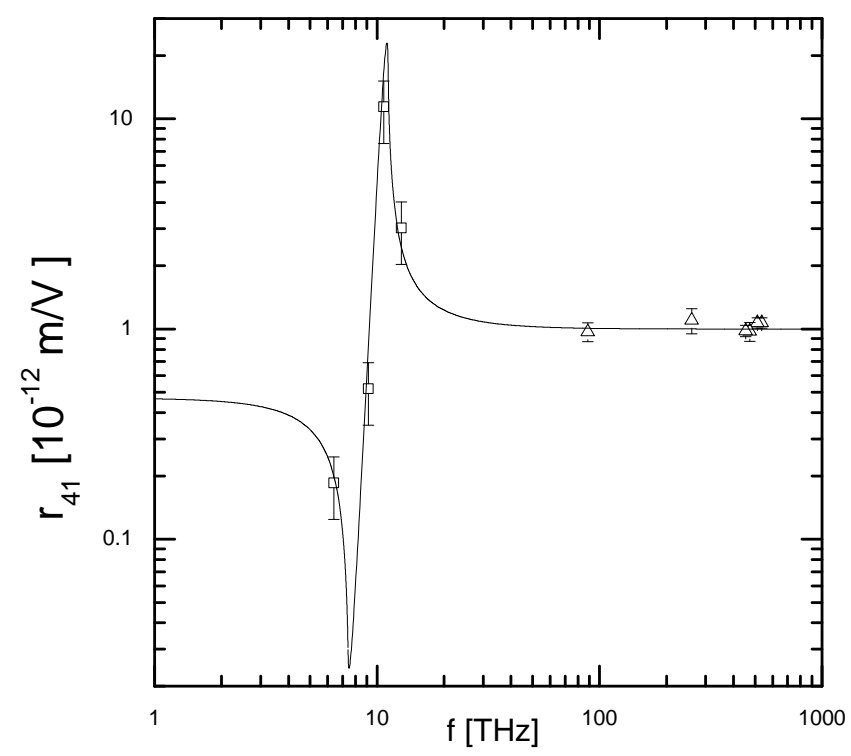

Figure 10: $r_{41}(f)$ for GaP. The square symbols $\square$ are experimental data taken from Ref. [19], the triangles $\triangle$ from Ref. [25]. The curve is a fit of the experimental data using Eq. (23) with the parameters shown in the text. 


\section{Propagation of the $\mathrm{THz}$ and Laser Pulses}

\subsection{Phase and group velocities}

The refractive index $n$ of $\mathrm{ZnTe}(\mathrm{GaP})$ decreases with increasing wavelength in the optical regime. In the $\mathrm{THz}$ region below the TO resonance $n$ increases with the frequency. The short $\mathrm{THz}$ and Ti:Sa laser pulses propagate with the group velocity

$$
v_{g}=\frac{c}{n}\left(1+\frac{\lambda}{n} \frac{d n}{d \lambda}\right)=\frac{c}{\left(n+f \frac{d n}{d f}\right)}
$$

which is in both cases lower than the phase velocity of the contributing harmonic waves. The phase and group velocities of ZnTe and GaP are shown in Fig. 11 as functions of frequency. For comparison also the optical group velocity at $\lambda=0.8 \mu \mathrm{m}$ is plotted. While at low frequency the $\mathrm{THz}$ pulse propagates with a somewhat higher speed than the laser pulse, there is a growing mismatch in the velocities when one approaches the TO resonance of of $5.3 \mathrm{THz}$ in $\mathrm{ZnTe}$ and $11 \mathrm{THz}$ in $\mathrm{GaP}$.
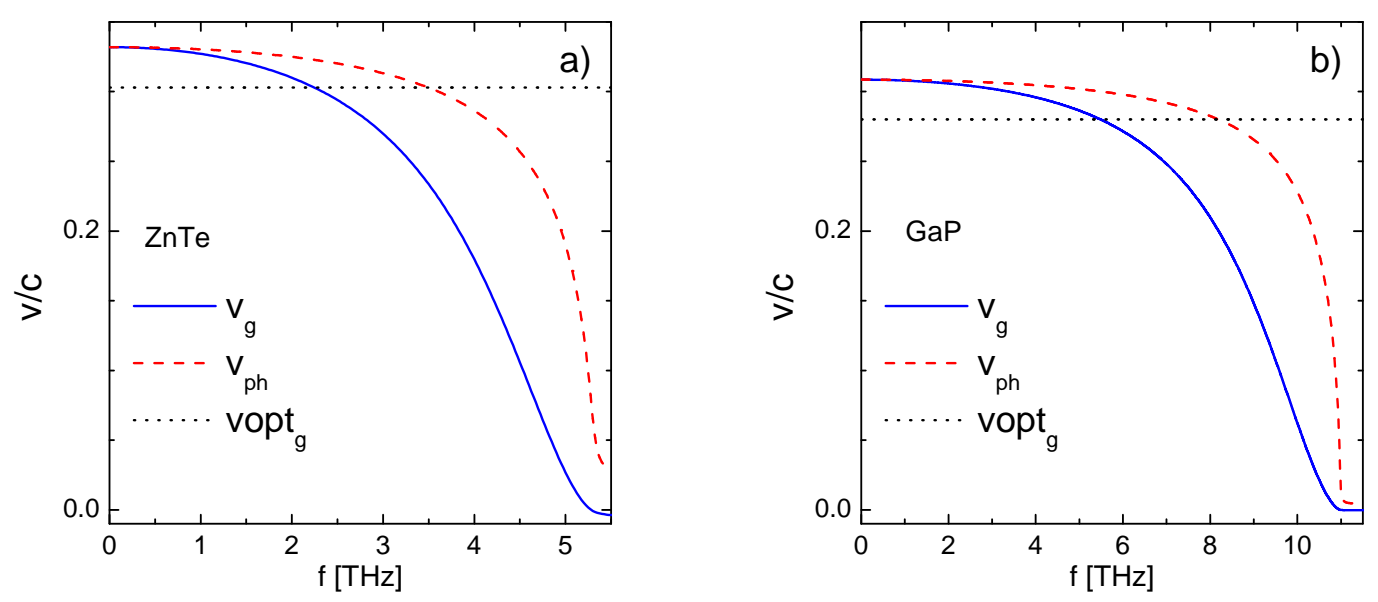

Figure 11: a) Phase velocity and group velocity of $\mathrm{ZnTe}$ in the $\mathrm{THz}$ range. The velocities have been divided by $c$. The group velocity of optical radiation at $0.8 \mu \mathrm{m}$ is shown as a dotted line. b) Phase and group velocity of GaP.

\subsection{Electro-optic response function}

For ideal electro-optic sampling conditions the $\mathrm{THz}$ pulse and the laser pulse should propagate at the same speed. The difference in speed leads to a reduced time resolution. It is customary to characterize the electro-optic efficiency by a response function which 
depends on the $\mathrm{THz}$ frequency $f$ and the crystal thickness $d$ :

$$
\begin{aligned}
G(f, d) & =\frac{2}{1+n(f)+i \kappa(f)} \frac{1}{d} \int_{0}^{d} \int_{-\infty}^{\infty} \exp \left[i(k z-2 \pi f t] \delta\left(z-v_{g} t\right) d t d z\right. \\
& =\frac{2}{1+n(f)+i \kappa(f)} \frac{1}{d} \int_{0}^{d} \exp \left[i 2 \pi f z\left(\frac{1}{v_{p h}(f)}-\frac{1}{v_{g}}\right)\right] d z
\end{aligned}
$$

where $v_{p h}(f)$ is the phase velocity at the THz frequency $f$ and $v_{g}$ the optical group velocity at the laser wavelength. The factor

$$
A_{\text {trans }}(f)=\frac{2}{1+n(f)+i \kappa(f)}
$$

is the frequency-dependent transmission coefficient for the transition of the $\mathrm{THz}$ electric field from vacuum into the EO crystal ${ }^{3}$. The EO response function of ZnTe is shown in Fig. 12 for different crystal thicknesses from $100 \mu \mathrm{m}$ to $1 \mathrm{~mm}$. It is obvious that high

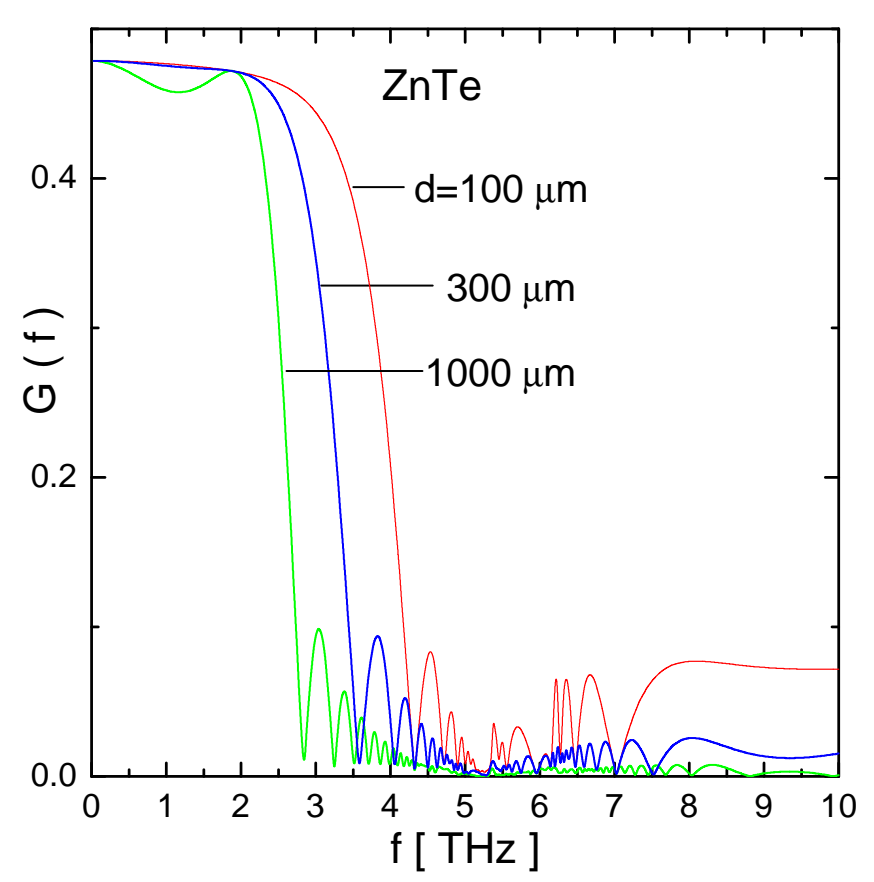

Figure 12: EO response function of ZnTe for crystal thickness of $100 \mu \mathrm{m}, 300 \mu \mathrm{m}$ and $1000 \mu \mathrm{m}$.

$\mathrm{THz}$ frequencies can only be reached in sufficiently thin crystals. The TO resonance sets an upper limit of about $4.5 \mathrm{THz}$ to the accessible frequency range.

Gallium phosphide permits to double the frequency range. From Fig. 13 one can see that a fairly thin crystal $(d \leq 100 \mu \mathrm{m})$ should be used to exploit this capability. At too large

\footnotetext{
${ }^{3}$ Some authors include the EO coefficient $r_{41}(f)$ in the definition of the response function, given then by $G(f, d) \cdot r_{41}(f)$. This is discussed in the appendix.
} 
a thickness, for example $d=300 \mu \mathrm{m}$, GaP reaches only about $3 \mathrm{THz}$ and is therefore inferior to ZnTe with its much larger EO coefficient $r_{41}$. The figures 12 and 13 suggest to use a ZnTe crystal thickness $d \approx 300 \mu \mathrm{m}$ as a compromise between high-frequency response and signal amplitude. For GaP the thickness should be about $100 \mu \mathrm{m}$ if one wants to detect signals with frequencies up to $10 \mathrm{THz}$. The small EO signal amplitude is then an unavoidable consequence. The simulations discussed below are mainly based on these crystal thicknesses.

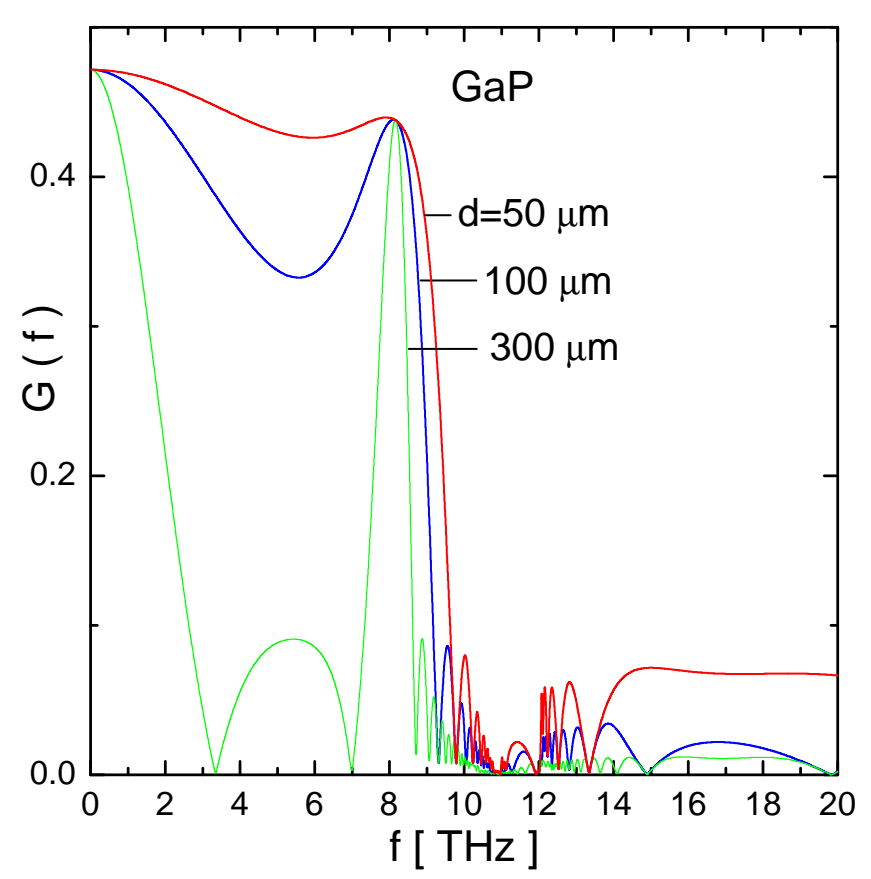

Figure 13: EO response function of GaP for a crystal thickness of $50 \mu \mathrm{m}, 100 \mu \mathrm{m}$ and $300 \mu \mathrm{m}$.

\subsection{Propagation and distortion of a Gaussian THz pulse in the EO crystal}

Instead of using the EO response function we prefer a more physical approach in which both the $\mathrm{THz}$ pulse and the optical laser pulse are propagated as wave packets through the EO crystal. The best insight into the physics of electro-optic sampling is provided by studying Gaussian bunches of well-defined width. Realistic bunch shapes will be discussed in Sect. 5. We use a cylindrical coordinate system $\left(r=\sqrt{x^{2}+y^{2}}, \theta, z\right)$ with the relativistic beam moving in the $z$ direction. The longitudinal charge distribution in the electron bunch is described by a Gaussian of variance $\sigma_{z}=c \sigma_{t}$. The line charge density is then

$$
\rho(z, t)=\frac{Q}{\sqrt{2 \pi} \sigma_{z}} \exp \left(-\frac{(z-c t)^{2}}{2 \sigma_{z}^{2}}\right)
$$


For highly relativistic electrons with a Lorentz factor $\gamma>1000$ the electric field of the bunch is concentrated in a flat disk perpendicular to the direction of motion. The field has mainly a radial component and can be written as

$$
E_{r}(r, z, t)=\frac{\rho(z, t)}{2 \pi \varepsilon_{0} r} .
$$

Let the EO crystal be located at $z=0$ at a distance $r_{0}$ from the beam axis. The electric field at the position of the crystal has the time dependence

$$
E_{r}(t)=E_{0} \exp \left(-\frac{t^{2}}{2 \sigma_{t}^{2}}\right) \quad \text { with } \quad E_{0}=\frac{Q}{2 \pi \varepsilon_{0} r_{0} \sqrt{2 \pi} c \sigma_{t}} .
$$

We call $F_{E}(f)$ the Fourier transform of the electric field pulse, which in this special case can be computed analytically or, for more complicated charge distributions, by an FFT (Fast Fourier Transform) algorithm. At the interface between the accelerator vacuum and the EO crystal, some fraction of the incident wave is reflected, the remaining part is transmitted into the dielectric crystal. The amplitude transmission coefficient depends on frequency and is given by the expression

$$
A_{\text {trans }}(f)=\frac{2}{n(f)+i \kappa(f)+1} .
$$

The Fourier component of the transmitted electric field pulse is

$$
F_{\text {trans }}(f)=F_{E}(f) \frac{2}{n(f)+i \kappa(f)+1} .
$$

To propagate the $\mathrm{THz}$ pulse inside the EO material we subdivide the crystal into ten thin slices of thickness $\delta=d / 10$. The Fourier component at slice $j$ is given by

$$
F_{\text {slice } j}(f)=F_{\text {trans }}(f) \exp \left(i \frac{2 \pi f}{c} n(f) d_{j}-\frac{2 \pi f}{c} \kappa(f) d_{j}\right)
$$

where $d_{j}=(j+0.5) \delta$ is the depth of slice $j$. The phase propagation is determined by the refractive index $n(f)$, the attenuation by the extinction coefficient $\kappa(f)$. The time profile of the pulse at slice $j$ is then simply obtained by applying the inverse FFT to Eq. (32):

$$
E_{j}^{T H z}(t)=I F F T\left[F_{\text {trans }}(f) \exp \left(i \frac{2 \pi f}{c} n(f) d_{j}-\frac{2 \pi f}{c} \kappa(f) d_{j}\right)\right] .
$$

As an illustration we show in Fig. 14 the time profile of the $\mathrm{THz}$ pulse at 10 positions inside a $300 \mu \mathrm{m}$ thick ZnTe crystal. One can easily see that the pulse width increases with increasing depth in the crystal, and that oscillations gradually develop. These high frequency oscillations lag behind the main pulse since the $\mathrm{THz}$ refractive index grows approaching the TO resonance at $5.3 \mathrm{THz}$ (see Fig. 7).

Also the Ti:Sa laser pulse changes its shape when it traverses the EO crystal because the optical refractive index has a nonlinear dependence on the wavelength, see Figures 5 and 6. Assuming a Gaussian distribution for the intensity

$$
I_{\text {las }}(t) \propto \exp \left(-\frac{t^{2}}{2 \sigma_{0}^{2}}\right)
$$



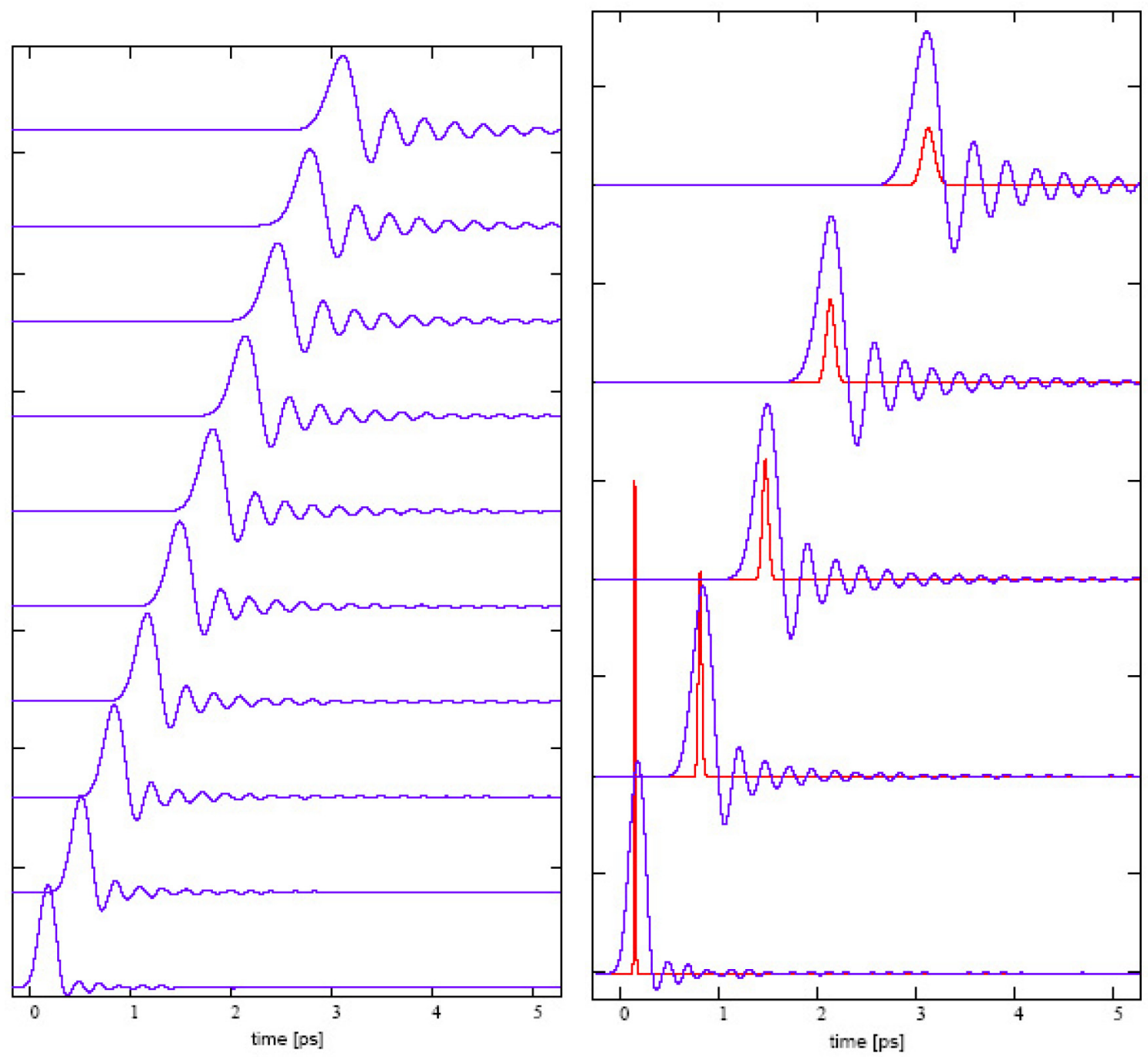

Figure 14: Left: The propagation of a Gaussian THz pulse with initial variance of $\sigma_{z}=20 \mu \mathrm{m}$ in a $300 \mu \mathrm{m}$ thick ZnTe crystal. Plotted are the time profiles in the ten $30 \mu \mathrm{m}$ thick slices of the crystal. Right: The propagation of a Gaussian THz pulse and of a Gaussian Ti:Sa laser pulse in a $300 \mu \mathrm{m}$ thick ZnTe crystal. Plotted are the time profiles at selected positions in the crystal.

with a bandwidth-limited FWHM (full width at half maximum) of $\Delta t=2 \sqrt{2 \ln 2} \sigma_{0}=15$ fs for the Ti:Sa laser (Femtosource COMPACT, Femtolasers, Vienna) used in our EOS experiment we expect a growth of the variance with increasing depth in the EO crystal [26]

$$
\sigma(z)=\sigma_{0} \sqrt{1+\left(\frac{z}{L_{\text {char }}}\right)^{2}}
$$

with a characteristic length [26]

$$
L_{c h a r}=\frac{\Delta t^{2}}{4 \ln 2 \frac{d}{d \omega}\left(v_{g}^{-1}\right)} .
$$

One gets $L_{c h a r}=30 \mu \mathrm{m}$ in ZnTe and $L_{\text {char }}=42 \mu \mathrm{m}$ in GaP. Figure 14 shows the $\mathrm{THz}$ and laser pulses at selected positions inside the $300 \mu \mathrm{m}$ thick ZnTe crystal. The laser 
pulse moves at a lower speed than the $\mathrm{THz}$ pulse ${ }^{4}$ and due to the nonlinear dispersion its width increases.

The detrimental effects of group velocity mismatch and pulse distortion are of course reduced by choosing a thinner crystal, but at the price of a lower detector signal. A quantitative analysis will be presented in the next section.

\subsection{Phase retardation and balanced detector signal}

\subsubsection{Without laser pulse broadening}

In the previous section we have computed the shape of the $\mathrm{THz}$ pulse in the 10 thin slices of the EO crystal and the relative timing with respect to the laser pulse ${ }^{5}$. Equation (16) will now be applied to calculate the retardation parameter $\Gamma$ for each slice. The angle between the electric vector of the THz field and the crystallographic axis $[-1,1,0]$ is chosen as $\alpha=0$. To be accurate, also the frequency dependence of the EO coefficient $r_{41}$ must be considered. This is done by multiplying the Fourier component of the propagated THz pulse (Eq. (32)) with $r_{41}(f)$, and by applying then the inverse FFT. The "effective" electric $\mathrm{THz}$ pulse in slice $j$ is therefore given by

$$
E_{j}^{e f f}(t)=I F F T\left[F_{\text {trans }}(f) \exp \left(i \frac{2 \pi f}{c} n(f) d_{j}-\frac{2 \pi f}{c} \kappa(f) d_{j}\right) \cdot r_{41}(f)\right]
$$

Since for ZnTe we disregard the frequency dependence of $r_{41}$ these pulses have the same shapes as shown in Fig. 14. In case of GaP the propagation of an "effective" Gaussian THz pulse (see Eq.(35)) with initial variance of $\sigma_{z}=10 \mu \mathrm{m}$ in a $100 \mu \mathrm{m}$ thick crystal is shown in Fig. 15. Approximating for the time being the laser pulse by a delta function we see immediately from the above figures that the phase retardation $\Gamma_{j}$ generated in slice $j$ is proportional to the electric field amplitude $E_{j}\left(t_{j, l a s e r}\right)$ at the arrival time of the laser pulse in slice $j$,

$$
\Gamma_{j}=\frac{2 \pi}{\lambda_{0}} n_{0}^{3} \delta E_{j}^{e f f}\left(t_{j, l a s e r}\right)
$$

This time is

$$
t_{j, \text { laser }}=d_{j} / v_{\text {group }}+\tau
$$

where we have allowed for a variable time delay $\tau$ between $\mathrm{THz}$ and laser pulse. The total phase retardation accumulated in the EO crystal can be computed as a sum over the contributions of each slice:

$$
\Gamma(\tau)=\sum_{j} \Gamma_{j}(\tau)=\frac{2 \pi}{\lambda_{0}} n_{0}^{3} \delta \sum_{j} E_{j}^{e f f}\left(d_{j} / v_{\text {group }}+\tau\right) .
$$

In the actual EOS experiment the delay $\tau$ is varied in small steps to scan the $\mathrm{THz}$ pulse.

\footnotetext{
${ }^{4}$ The frequency components of a $\mathrm{THz}$ pulse with $\sigma_{z}=20 \mu \mathrm{m}$ are predominantly below 2 THz. According to Fig. 11 the $\mathrm{THz}$ pulse has therefore a higher group velocity than the laser pulse.

${ }^{5}$ The slice thickness has to be chosen small enough, so that the shift between $\mathrm{THz}$ and laser pulse within a slice can be neglected. Increasing the number of slices from 10 to 20 has a negligible effect on the computed EO signal.
} 

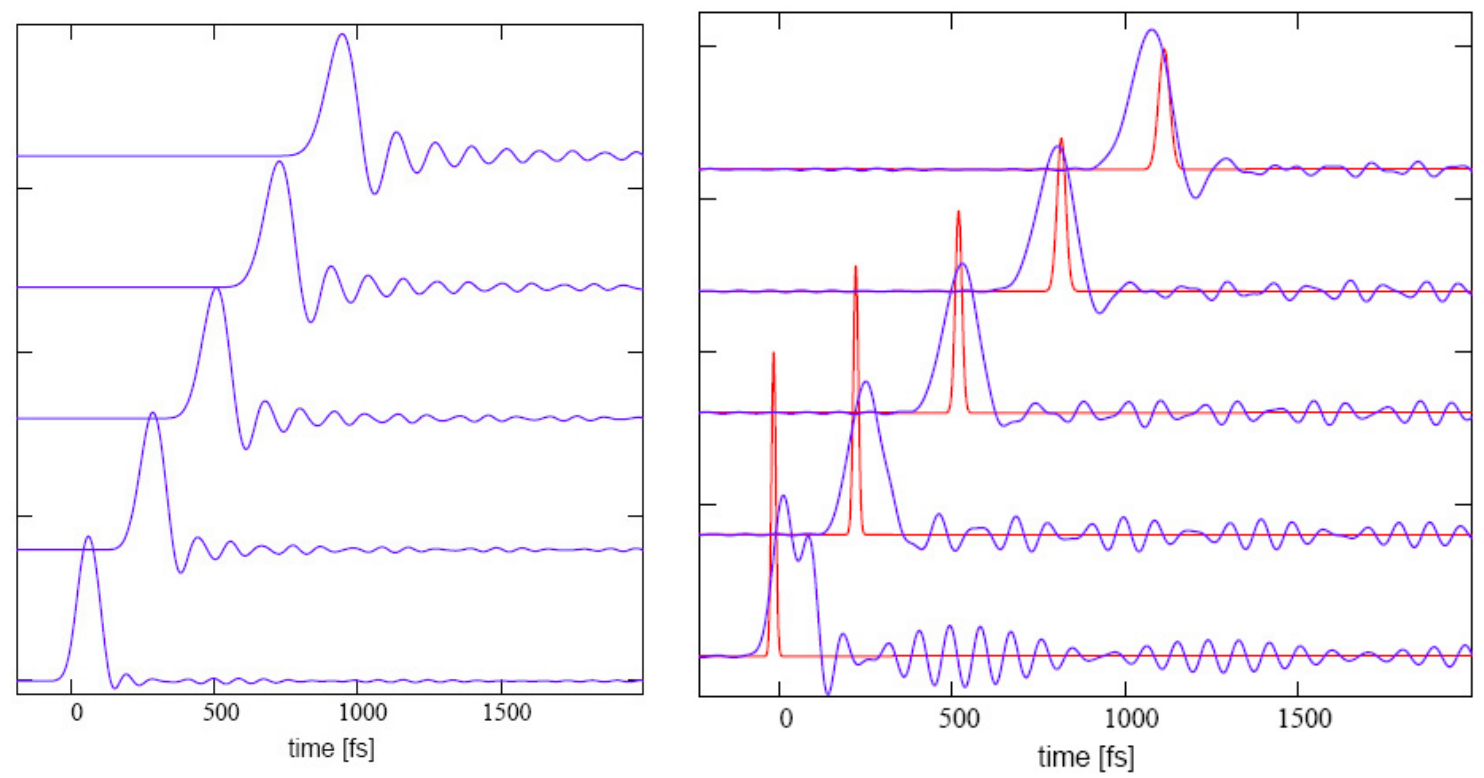

Figure 15: Left: The propagation of a Gaussian THz pulse (variance $\sigma_{z}=10 \mu \mathrm{m}$ ) in a $100 \mu \mathrm{m}$ thick GaP crystal at selected positions. Right: Same as in the left plot, but the frequency dependence of $r_{41}$ is taken into account according to Eq.( 35). Shown is also the broadening of a Gaussian Ti:Sa laser pulse.

\subsubsection{With laser pulse broadening}

The next refinement is to incorporate the laser pulse broadening. According to Eq. (34) the rms width of the laser pulse in slice $j$ is:

$$
\sigma_{j}=\sigma_{0} \sqrt{1+\left(\frac{d_{j}}{L_{\text {char }}}\right)^{2}}
$$

The overlap of the $\mathrm{THz}$ and laser pulses in each slice is computed by a convolution integral.

$$
\Gamma(\tau)=\frac{2 \pi}{\lambda_{0}} n_{0}^{3} \delta \sum_{j}\left[\int E_{j}^{e f f}\left(d_{j} / v_{\text {group }}+t\right) \frac{1}{\sqrt{2 \pi} \sigma_{j}} \exp \left(-\frac{(t-\tau)^{2}}{2 \sigma_{j}^{2}}\right) d t\right] .
$$

The signal in the balanced diode detector is proportional to $\sin \Gamma(\tau)$, see Eq. (17). In Fig. 16 we show the expected balanced diode detector signal as a function of the relative delay $\tau$ between the $\mathrm{THz}$ and the laser pulse. The computations have been made for a $500 \mu \mathrm{m}$ thick ZnTe crystal and a THz pulse with $\sigma_{z}=20 \mu \mathrm{m}$. The influence of laser pulse broadening in the rather thick EO crystal is visible but not very significant. For thinner crystals it can be neglected so it is legitimate to use Eq. (??) to compute the phase retardation parameter $\Gamma$.

\section{Quality of Bunch Shape Determination by EOS}

In this section we assume various longitudinal profiles for the electron bunches: Gaussian, Gaussian with exponential tail, sum of two Gaussians, and study how well the initial 


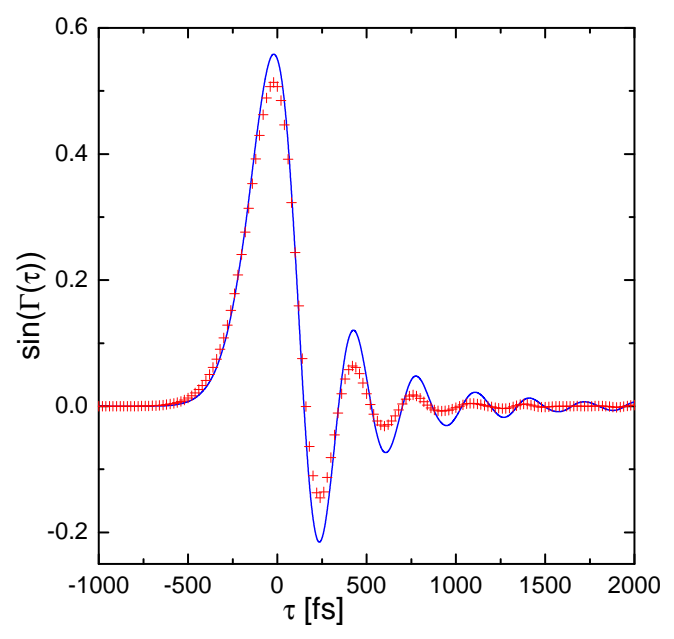

Figure 16: Signal in the balanced diode detector for a $500 \mu \mathrm{m}$ thick ZnTe crystal which is placed at a distance $r_{0}=14 \mathrm{~mm}$ from the electron beam. Bunch charge $Q=0.2 \mathrm{nC}$, rms bunch length $\sigma_{z}=20 \mu \mathrm{m}$. Plotted is $\sin \Gamma(\tau)$ as a function of the relative delay $\tau$ between the $\mathrm{THz}$ and the laser pulse. Continuous blue curve: delta-function like laser pulse, Eq. (??). Crosses: laser pulse broadening taken into consideration, Eq. (37).

charge distribution can be recovered by the EO sampling technique. As mentioned above, the electrons are taken as highly relativistic so that the electromagnetic field pulse impinging on the EO crystal has essentially the same time profile as the charge density in the bunch.

\subsection{Gaussian bunch shape}

\subsubsection{Pulse broadening}

The analysis procedure for a Gaussian $\mathrm{THz}$ pulse has been explained in the previous section. Here we summarize the simulation results for $\mathrm{ZnTe}$ and $\mathrm{GaP}$ crystals for $\mathrm{THz}$ pulses of various width. The bunch charge is chosen as $Q=0.5 \mathrm{nC}$, the distance between the electron beam and the spot on the EO crystal which is scanned by the laser is taken as $r_{0}=14 \mathrm{~mm}$. First we look at a rather thick ZnTe crystal with $d=300 \mu \mathrm{m}$ and compare a long $\mathrm{THz}$ pulse with $\sigma_{z}=100 \mu \mathrm{m}$ and a short pulse with $\sigma_{z}=20 \mu \mathrm{m}$. The long pulse traverses the ZnTe crystal with almost unmodified shape while the short pulse suffers a significant broadening and shape distortion, see Fig. 17. The expected signal in the balanced detector, proportional to $\sin (\Gamma)$, is compared in Fig. 18 with the original $\mathrm{THz}$ pulse shape. In the case of a long pulse the detector signal reproduces the Gaussian charge distribution very well. For the short bunch, however, the FWHM of the main peak is a factor of 1.9 larger. Moreover, the main peak is followed by oscillations whose frequency spectrum is peaked at around $4 \mathrm{THz}$, i.e. in the region where the refractive index shows a strong rise towards the TO resonance at $5.3 \mathrm{THz}$.

Now we study a GaP crystal of $100 \mu \mathrm{m}$ thickness. The balanced detector signal expected from a pulse with $\sigma_{z}=20 \mu \mathrm{m}$ is shown in Fig. 19. The original pulse shape is almost 

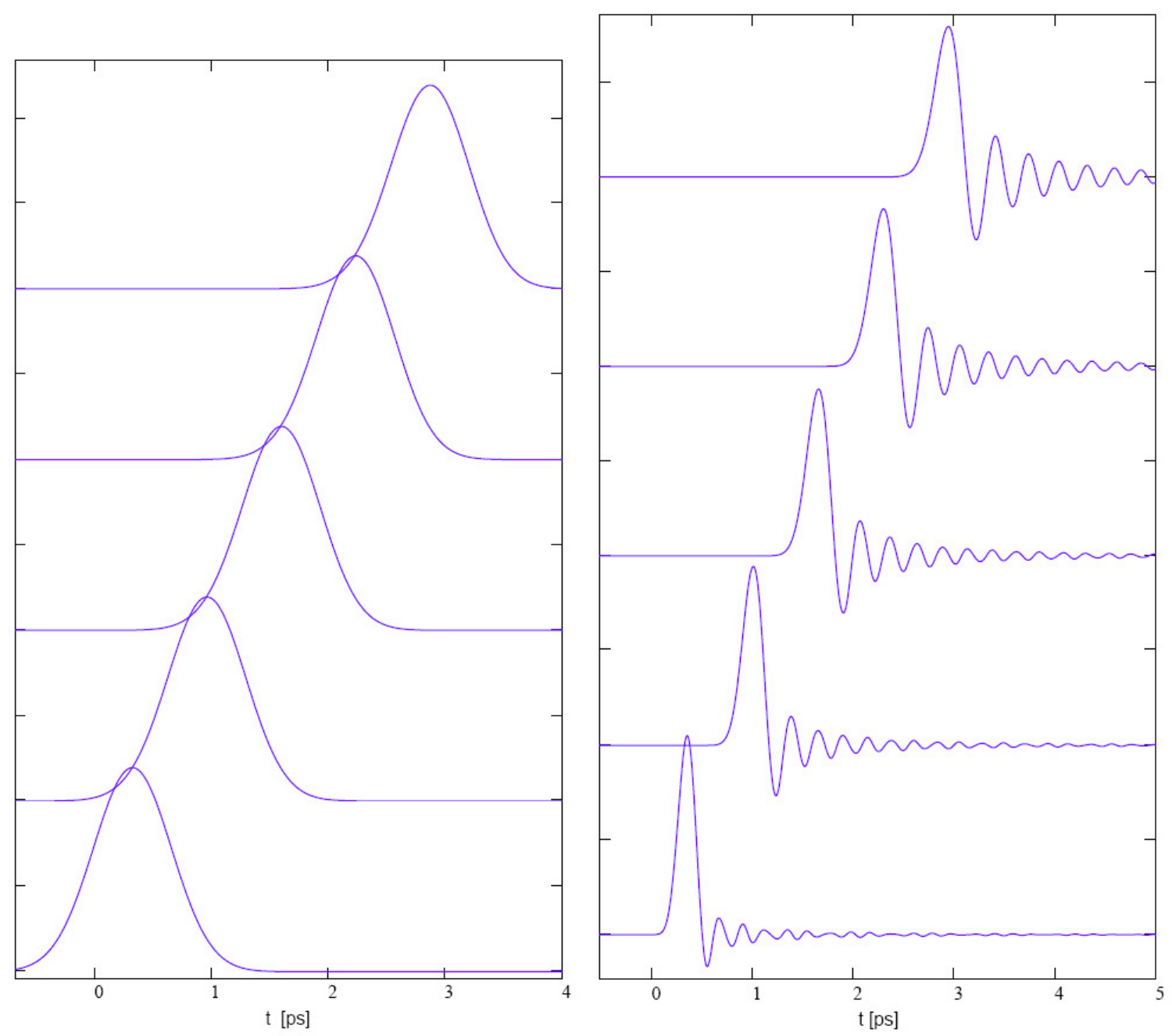

Figure 17: Left: The shape of a long THz pulse $\left(\sigma_{z}=100 \mu \mathrm{m}\right)$ at selected positions in a $300 \mu \mathrm{m}$ thick ZnTe crystal. Right: The shape of a short pulse $\left(\sigma_{z}=20 \mu \mathrm{m}\right)$ at the same positions.

reproduced, the broadening factor amounts to only 1.15. Note that no oscillations are observed in the tail of the detector signal. This is easily understood since the $20 \mu \mathrm{m}$ bunch has very small Fourier components near the TO resonance of GaP at $11 \mathrm{GHz}$. A shorter bunch, however, leads to oscillations in the detector signal, and a significant pulse stretching (the FWHM is a factor 1.6 wider), see Fig. 19.

In Fig. 20a we show the broadening factor as a function of the rms bunch length for $\mathrm{ZnTe}$ and GaP. For rms THz pulse lengths above $30 \mu \mathrm{m}$ both EO materials are suited to reconstruct the shape without distortion. It is evident that GaP permits the measurements of far shorter pulses than ZnTe. However, bunch lengths with $\sigma_{z} \leq 10 \mu \mathrm{m}$ cannot be resolved even with a GaP EO crystal. The FWHM of the EO signal is plotted in Fig. 20b as a function of the FWHM of the incoming pulse. It is evident that the lowest measurable FWHM is $200 \mathrm{fs}$ in ZnTe and $100 \mathrm{fs}$ in GaP. Note that we have neglected here any time jitter between the $\mathrm{THz}$ and laser pulses. 

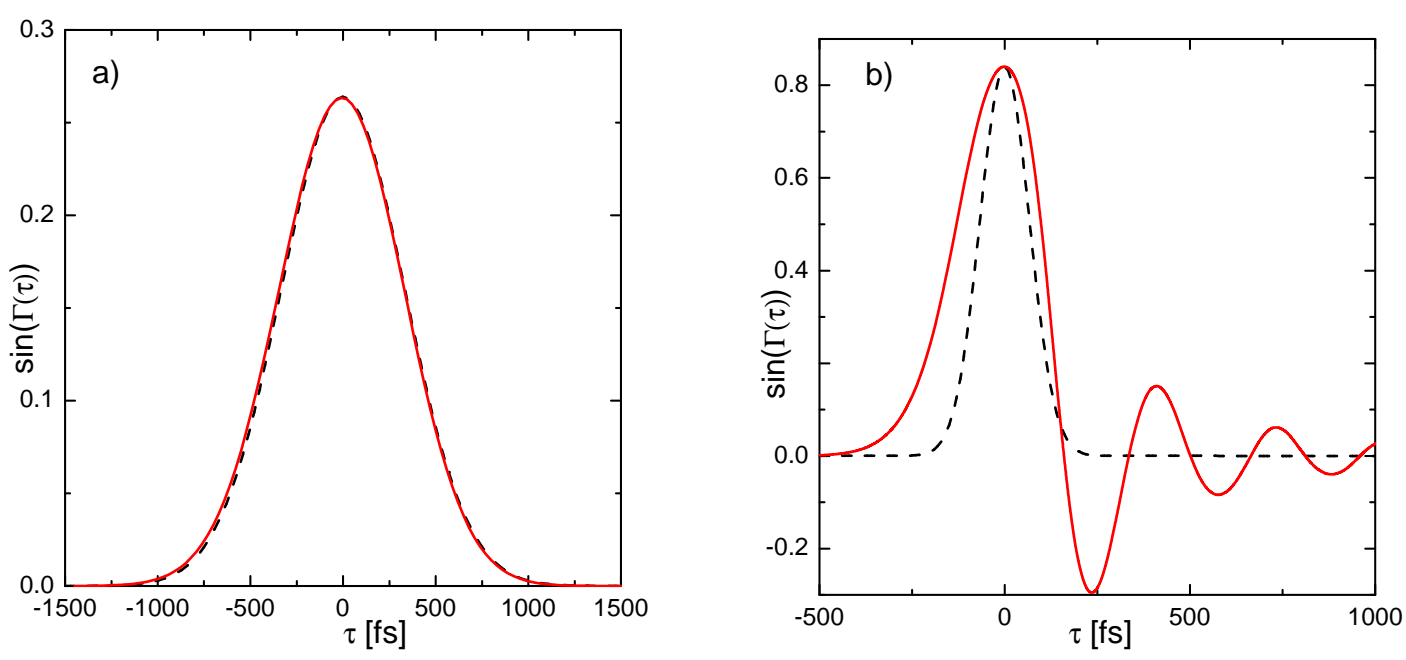

Figure 18: ZnTe crystal, $d=300 \mu \mathrm{m}$. a) The balanced-detector signal (solid curve) of a long THz pulse $\left(\sigma_{z}=100 \mu \mathrm{m}\right)$ in comparison with the original pulse shape (dashed curve). b) The balanced-detector signal (solid curve) of a short $\mathrm{THz}$ pulse $\left(\sigma_{z}=20 \mu \mathrm{m}\right)$ compared to the original pulse shape (dashed curve).
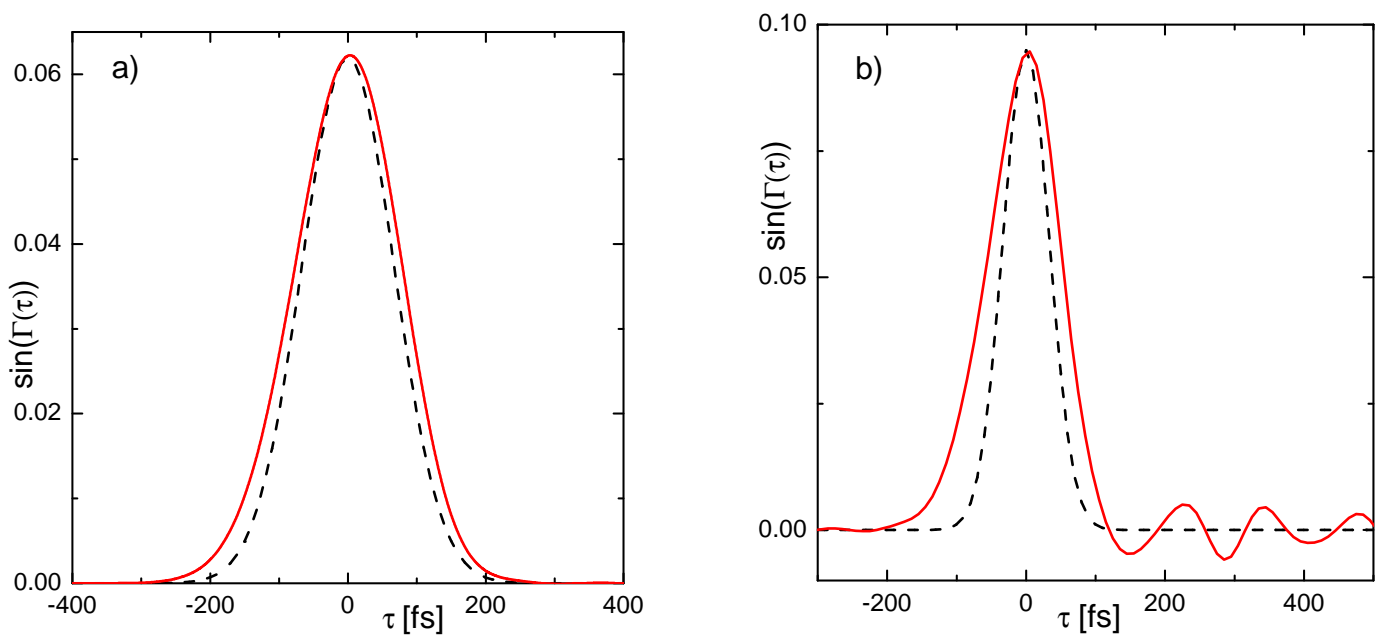

Figure 19: GaP crystal, $d=100 \mu \mathrm{m}$. a) The balanced-detector signal (solid curve) of a $\left(\sigma_{z}=20 \mu \mathrm{m}\right) \mathrm{THz}$ pulse in comparison with the original pulse shape (dashed curve). b) The balanced-detector signal (solid curve) of a shorter $\mathrm{THz}$ pulse $\left(\sigma_{z}=10 \mu \mathrm{m}\right)$ compared to the original pulse shape (dashed curve).

\subsubsection{Limits on the time resolution}

For short bunches GaP is superior to ZnTe owing to the factor of two higher TO frequency. An important question is how far one can go down in bunch length and still get a reliable EO signal. An astonishing result of our calculations is that decreasing the GaP crystal 

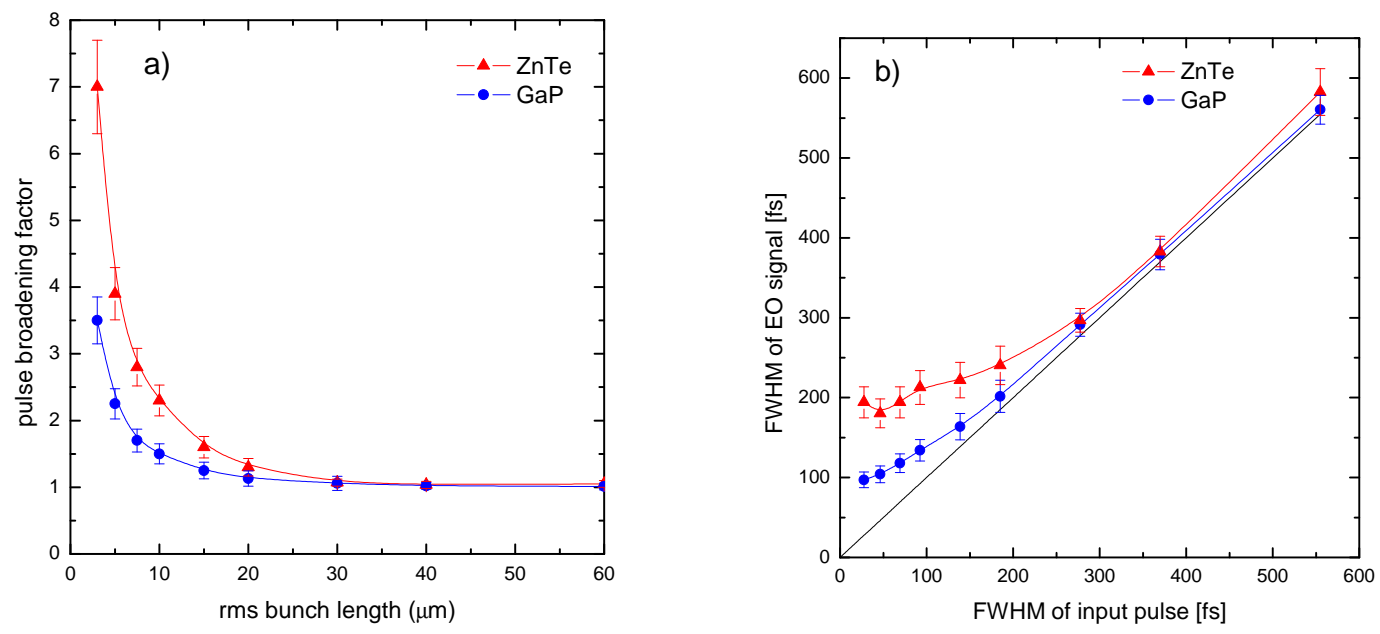

Figure 20: Pulse broadening in ZnTe (triangles) and in GaP (circles). The EO crystal has a thickness $100 \mu \mathrm{m}$. a) Pulse broadening factor as a function of the initial rms bunch length. b) FWHM of the EO signal as a function of the FWHM of the electron bunch.

thickness helps only very little to improve the resolution for bunches with $\sigma_{z} \leq 10 \mu \mathrm{m}$. This is illustrated by Fig. 21 where we compare the incoming THz pulse shape (in vacuum) with the $\mathrm{THz}$ pulse at a depth of only $5 \mu \mathrm{m}$ in the GaP crystal. Already at such

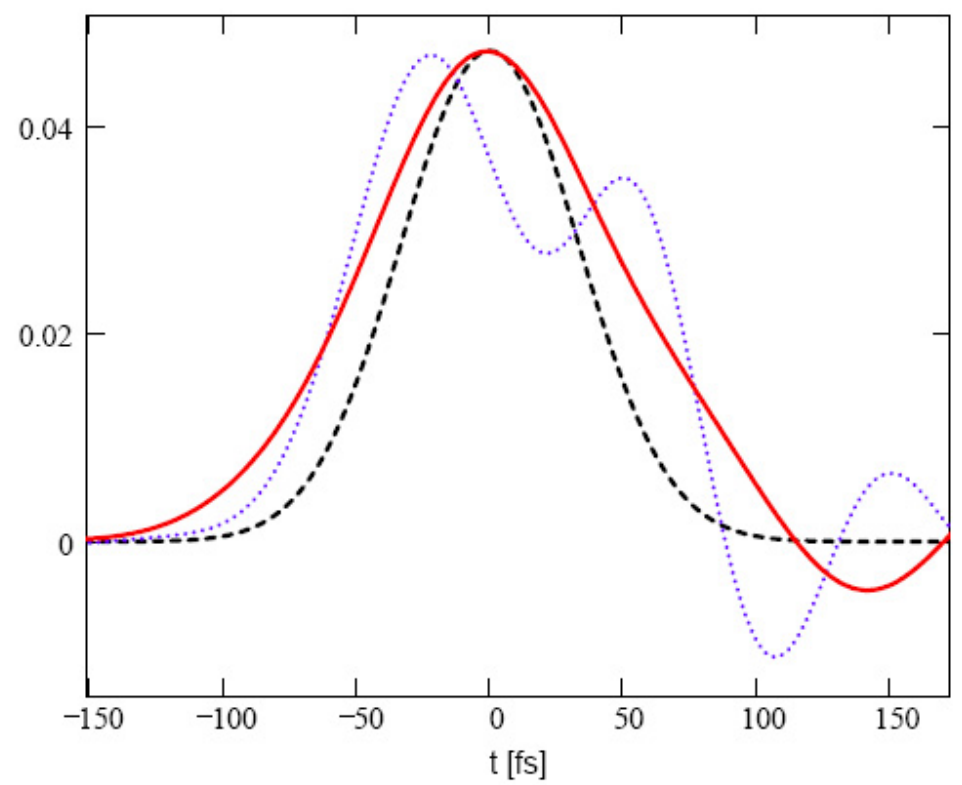

Figure 21: GaP crystal of $50 \mu \mathrm{m}$ thickness. The incident pulse with $\sigma_{z}=10 \mu \mathrm{m}$ is shown as a dashed curve. Dotted curve: shape of the THz pulse at a depth of $5 \mu \mathrm{m}$. Solid curve: balanced detector signal. 
a small depth the propagated $\mathrm{THz}$ pulse has acquired a pronounced oscillatory structure and is significantly wider than the incident pulse. In fact, it has about the same width as the balanced detector signal produced by the entire $50 \mu \mathrm{m}$ thick GaP crystal, see Fig. 21 . This calculation shows that a reduction of the crystal thickness far below $100 \mu \mathrm{m}$ yields only a marginal improvement in time resolution but has the disadvantage of a lower signal amplitude.

The TO resonance puts indeed a rather severe limit on the shortest pulse length which can be resolved. Figure 22 shows the propagation of extremely short $\mathrm{THz}$ pulses in GaP. One observes very strong oscillations, especially in the first few microns of the crystal, and the original pulse shape is hardly discernable. This indicates that rms bunch lengths below $10 \mu \mathrm{m}$ are basically inaccessible with $\mathrm{GaP}$ as an electro-optic sensor, and even less so with ZnTe. The oscillations are generated by resonant amplification of the Fourier compononents of the bunch in the vicinity of the TO crystal oscillation frequency. A remarkable observation is that the strongest oscillations are introduced by the EO coefficient $r_{41}(f)$.
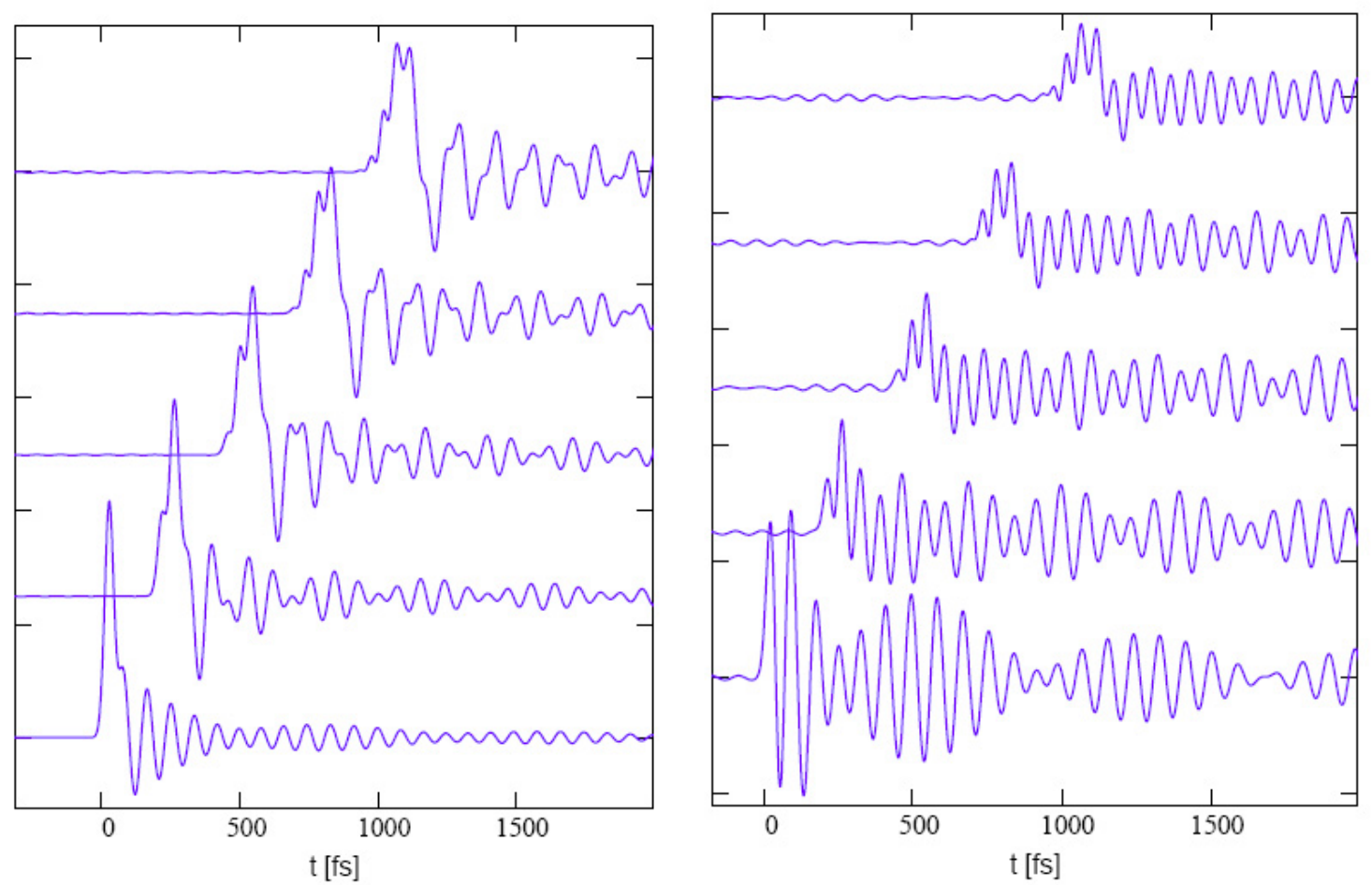

Figure 22: Extremely short pulses in a GaP crystal of $100 \mu \mathrm{m}$ thickness. Left: Shape of a Gaussian $\mathrm{THz}$ pulse with initial $\sigma_{z}=5 \mu \mathrm{m}$ at selected positions in the crystal. Right: Shape of the "effective" pulse, taking into account the contribution of $r_{41}(f)$ according to Eq. (35).

\subsubsection{Dependence of the EO signal on the crystal thickness}

Ideally the amplitude of the EO signal should be directly proportional to the thickness $d$ of the EO crystal. In reality, the $\mathrm{THz}$ pulse distortion leads to a slower than linear rise of 
the balanced detector signal with $d$ and eventually to a saturation. This is demonstrated in Fig. 23. Another disadvantage is that the FWHM of the signal grows considerably
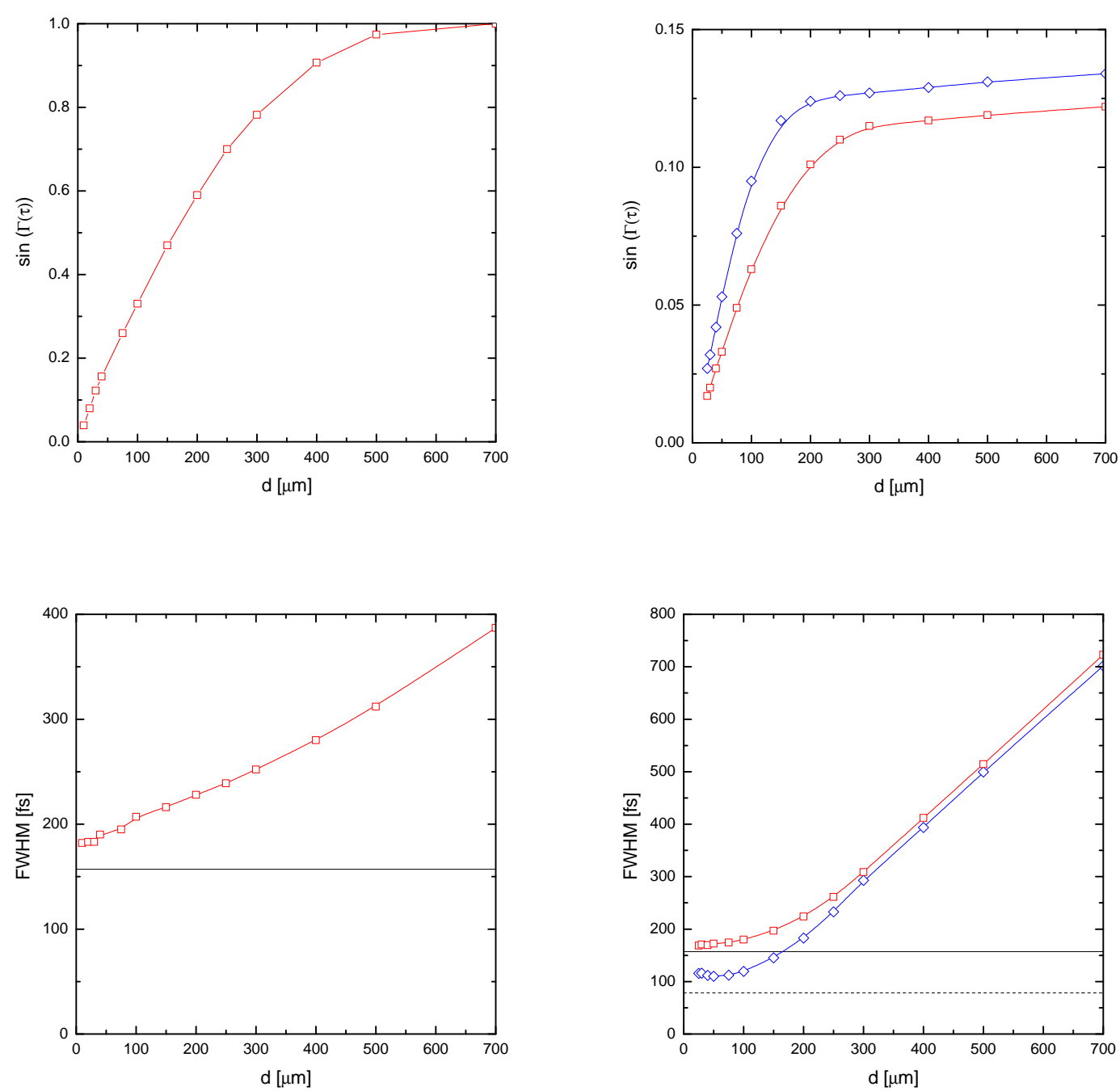

Figure 23: Dependence of the electro-optic signal on the thickness $d$ of the EO crystal. Top graphs: signal amplitude $\sin (\Gamma)$ vs. the thickness $d$. Left: a pulse with $\sigma_{z}=20 \mu \mathrm{m}$ in ZnTe; right: a pulse with $\sigma_{z}=20 \mu \mathrm{m}$ (squares) and with $\sigma_{z}=10 \mu \mathrm{m}$ (diamonds) pulse in GaP. Bottom graphs: FWHM of the detector signal as a function of $d$. Left: ZnTe, right: GaP. The FWHM of the incident pulse is shown as a solid line $\left(\sigma_{z}=20 \mu \mathrm{m}\right)$ and as a dashed line $\left(\sigma_{z}=10 \mu \mathrm{m}\right)$.

towards larger thickness. In order to preserve a good time resolution the crystal thickness should therefore not exceed $d=100 \mu \mathrm{m}$ by a large factor. On the other hand, going to $d=50 \mu \mathrm{m}$ or less does not really improve the resolution but only leads to much smaller signals. 


\subsection{Other bunch shapes}

In Figure 24 we compare the balanced-detector signal from a $100 \mu \mathrm{m}$ thick ZnTe resp. $\mathrm{GaP}$ crystal for a pulse shape that is expected in the present bunch compression scheme at the linac of the VUV FEL. The pulse consists of a Gaussian $\left(\sigma_{z}=20 \mu \mathrm{m}\right)$ with an exponential tail $[27,28]$ :

$$
E(t)= \begin{cases}\exp \left(-\frac{t^{2}}{2 \sigma_{t}^{2}}\right) & \text { for } t<t_{1} \\ \frac{A \exp \left(-t / \tau_{1}\right)}{\sqrt{\left(t+t_{0}\right) / \tau_{1}}} & \text { for } t>t_{1}\end{cases}
$$

where the following parameters are used:

$$
A=0.47, \sigma_{t}=66.7 \mathrm{fs}, t_{1}=8.9 \mathrm{fs}, \tau_{1}=500 \mathrm{fs}, t_{0}=100 \mathrm{fs} .
$$

As shown in Fig. 25 we have also studied the response of $100 \mu \mathrm{m}$ thick $\mathrm{ZnTe}$ and GaP
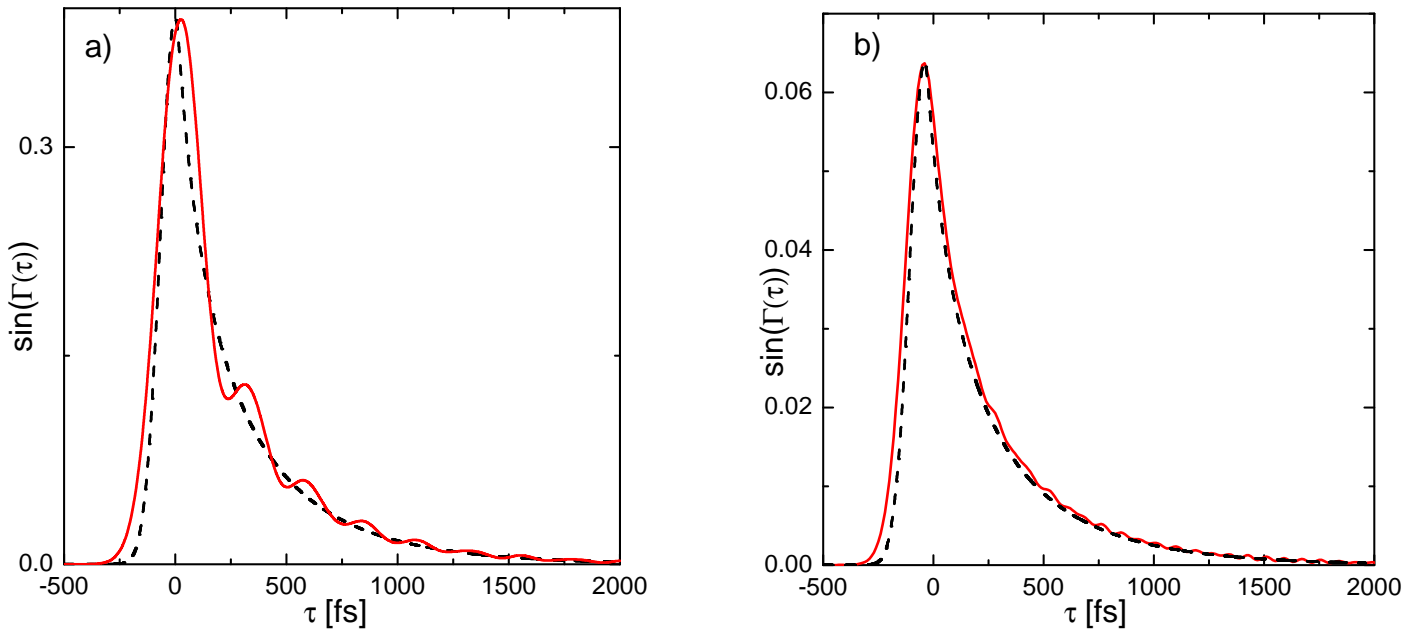

Figure 24: A Gaussian bunch with exponential tail from Eq.(38) (dashed curve) and its reconstruction (solid curve) in an EO experiment with a $100 \mu \mathrm{m}$ thick ZnTe (a) and GaP (b) crystal.

crystals for a double Gaussian given by:

$$
E(t)=\exp \left(-\frac{t^{2}}{2 \sigma_{t}^{2}}\right)+A \exp \left(-\frac{\left(t-t_{1}\right)^{2}}{2 t_{0}^{2}}\right)
$$

with the following parameters:

$$
A=0.2, \quad \sigma_{t}=66.7 \mathrm{fs}, \quad t_{1}=200 \mathrm{fs}, t_{0}=500 \mathrm{fs} .
$$

For both bunch shapes the signal is far better reproduced with GaP instead of ZnTe as an EO sensor. The oscillations on the tail which are prominent in the ZnTe data are largely suppressed using GaP because of the larger TO resonance frequency of $11 \mathrm{THz}$ compared to $5.3 \mathrm{THz}$ in ZnTe. 

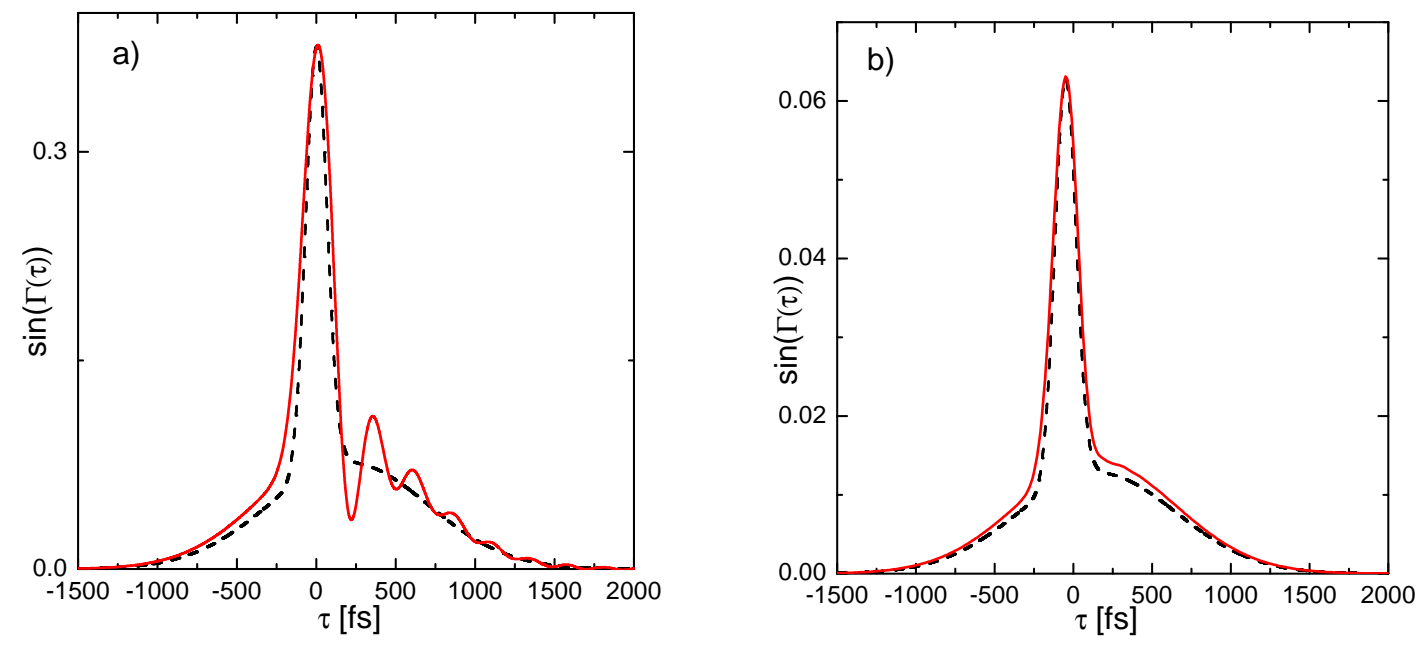

Figure 25: A double Gaussian bunch according to Eq.(39) (dashed curve) and its reconstruction (solid curve) in an EO experiment with a $100 \mu \mathrm{m}$ thick EO crystal. (a) ZnTe, (b) GaP.

\subsection{Multiple reflections in the EO crystal}

The $\mathrm{THz}$ pulse can undergo a reflection at the exit surface of the crystal, move back, and after a second reflection at the front surface, move again through the crystal in forward direction. This is sometimes referred to as the Fabry-Perot effect. The double-reflected pulse will be scanned by the laser if the laser pulse is delayed by the travel time $2 d / v_{T H z}$. The Fourier transform of the double-reflected $\mathrm{THz}$ pulse, just behind the front surface of the EO crystal, is given by

$$
F_{\text {double }}(f)=F_{\text {trans }}(f) A_{\text {ref }}^{2} \exp \left(i \frac{2 \pi f}{c} n(f)(2 d)-\frac{2 \pi f}{c} \kappa(f)(2 d)\right),
$$

where $A_{\text {ref }}=[1-n(f)-i \kappa(f)] /[1+n(f)+i \kappa(f)]$ is the amplitude reflection coefficient. These Fourier components are propagated to the ten slices of the crystal in the same manner as was applied for the direct $\mathrm{THz}$ pulse, see Sect. 3. The phase retardation signal in Fig. 26 shows the direct signal, and after the delay of $2 d / v_{T H z}$, the signal from the double-reflected $\mathrm{THz}$ pulse. This second signal is much weaker, about $20 \%$ of the first peak, mainly due to the reflection coefficient which enters quadratically. The next reflection will produce a pulse at twice the delay but with a signal amplitude of only a few per cent of the main peak. This and even higher-order reflections will be easily lost in the noise, so we refrain from summing up the Fabry-Perot effect in a geometric series. Also the laser pulse will undergo multiple reflections in the EO crystal. If the doublereflected laser pulse coincides with the double-reflected $\mathrm{THz}$ pulse one gets a contribution to the balanced detector signal at the position of the first main peak. This contribution is small since not only the $\mathrm{THz}$ pulse but also the laser pulse is attenuated by the double reflection. The effect can be avoided altogether if one uses a wedge-shaped EO crystal. The EO crystal then acts like a prism and deflects the direct laser beam by an angle of $(n-1) \alpha$ where $\alpha$ is the wedge angle and $n$ the refractive index for laser light. The 


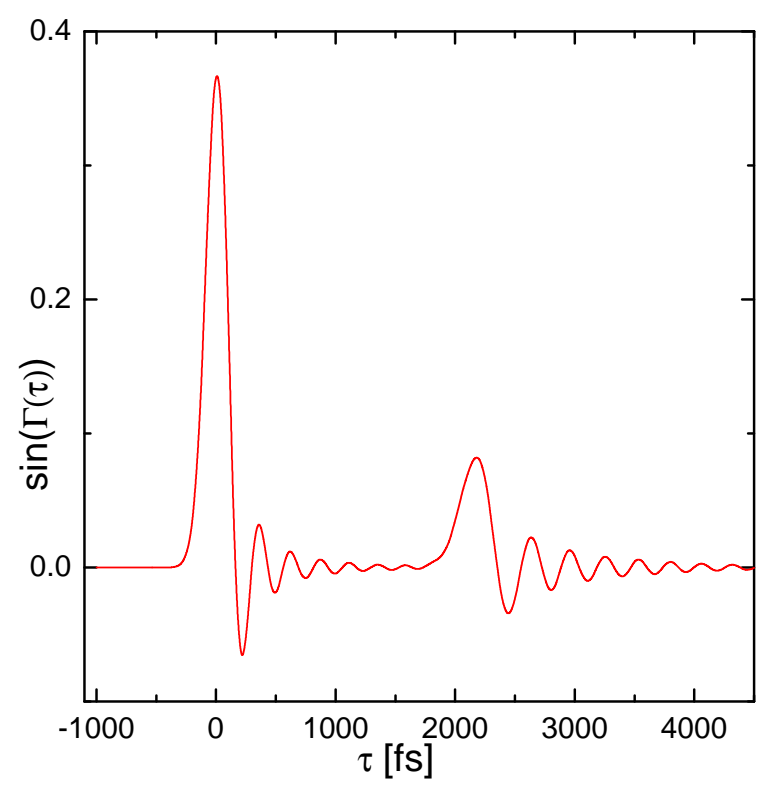

Figure 26: The balanced detector signal of a Gaussian THz pulse with $\sigma_{z}=20 \mu \mathrm{m}$ in a $100 \mu \mathrm{m}$ thick ZnTe crystal. Both the direct and the double-reflected pulse are shown.

double-reflected laser beam will leave the EO crystal at a deflection angle of $(3 n-1) \alpha$ and can therefore be easily separated from the direct laser beam. Note, however, that the wedge angle does not eliminate the multiple reflections of the $\mathrm{THz}$ pulse. The reason is that the electric field carried by the electron bunch is not well collimated like the Ti:Sa laser beam, but extends on the whole EO crystal.

\section{Summary}

Using the available experimental data on the material properties of $\mathrm{ZnTe}$ and $\mathrm{GaP}$ (frequency-depenent complex refractive index and electro-optic coefficient) we have studied the effects of pulse broadening, pulse shape distortion and group velocity mismatch in EOS experiments. Only the standard case has been considered where both the $\mathrm{THz}$ and the femtosecond laser pulse impinge perpendicular to the surface of the EO crystal. Our conclusion is that the shortest pulse length which can be recovered without significant distortion amounts to $200 \mathrm{fs}$ (FWHM) in ZnTe and to $100 \mathrm{fs}$ in GaP. Any time jitter between the $\mathrm{THz}$ and the laser pulse has been disregarded. In this paper we have not considered more advanced EO techniques such as temporal decoding [6] which permit high-resolution single-shot electron bunch diagnostics.

We want to thank Frank Ludwig for useful discussions. 


\section{Appendix A}

\section{Influence of Parameters Uncertainties on the EO Signals}

In the literature, as seen in section 2, there are differences up to few percent in the values of the material parameters needed for the simulations of the EO signal. In this section we investigate the effect of these uncertainties on the signal shape. By using the following parameters for ZnTe [9]:

$$
\text { ZnTe }: \varepsilon_{e l}=6.5, \quad f_{0}=5.35 \mathrm{THz}, \quad S_{0}=2.0, \quad \Gamma_{0}=0.09 \mathrm{THz}
$$

for a crystal with thickness $d=100 \mu \mathrm{m}$ and a Gaussian THz pulse with $\sigma_{z}=10 \mu \mathrm{m}$ we obtain the balanced detector signal shown in Fig. 27. For comparison the signal obtained with the parameters used in the previous sections [14] is also shown. A difference
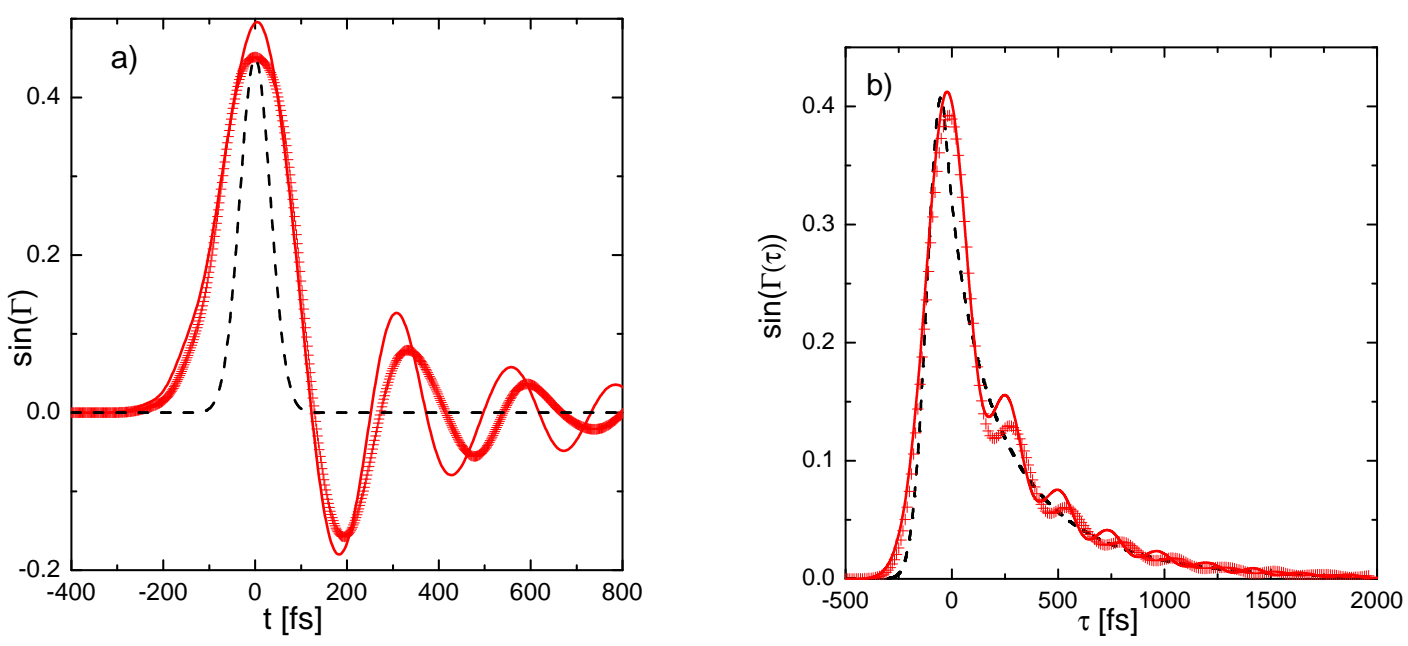

Figure 27: ZnTe crystal, $d=100 \mu \mathrm{m}$. a) The balanced detector signal of a Gaussian THz pulse $\left(\sigma_{z}=10 \mu \mathrm{m}\right)$ obtained with the parameters from Ref. [9] (solid curve) and with the ones from Ref. [14] (crosses). Shown is also the original pulse shape (dashed curve). b) The balanceddetector signal of a THz pulse with an initial shape given by Eq. (38) (dashed curve), computed for the parameter sets [9] (solid curve) and [14] (crosses).

of $7 \%$ is observed in the FWHM of the balanced detector signals. For longer bunches with $\sigma_{z}>15 \mu \mathrm{m}$ no appreciable difference is observed. The comparison has been also performed with the bunch shape given by Eq. (38). The FWHM of the signal of the balanced detector obtained with the two different sets of parameters differs by less than $2 \%$. 


\section{Appendix B}

\section{$\mathrm{THz}$ pulse reconstruction with the electro-optic response func- tion}

The total phase retardation can be computed from the EO response function (Figs. 12, 13) in the following way:

$$
\Gamma(\tau)=\frac{2 \pi}{\lambda_{0}} n_{0}^{3} \delta \cdot I F F T\left[F_{E}(f) G(f, d) r_{41}(f)\right] .
$$

A comparison with the pulse propagation method is made in Fig. 28. There is very good agreement at small crystal thickness ${ }^{6}$. Differences are only visible for thick crystals, and these are mainly caused by the laser pulse broadening which has been taken into account in the pulse propagation method but not in the response function method.
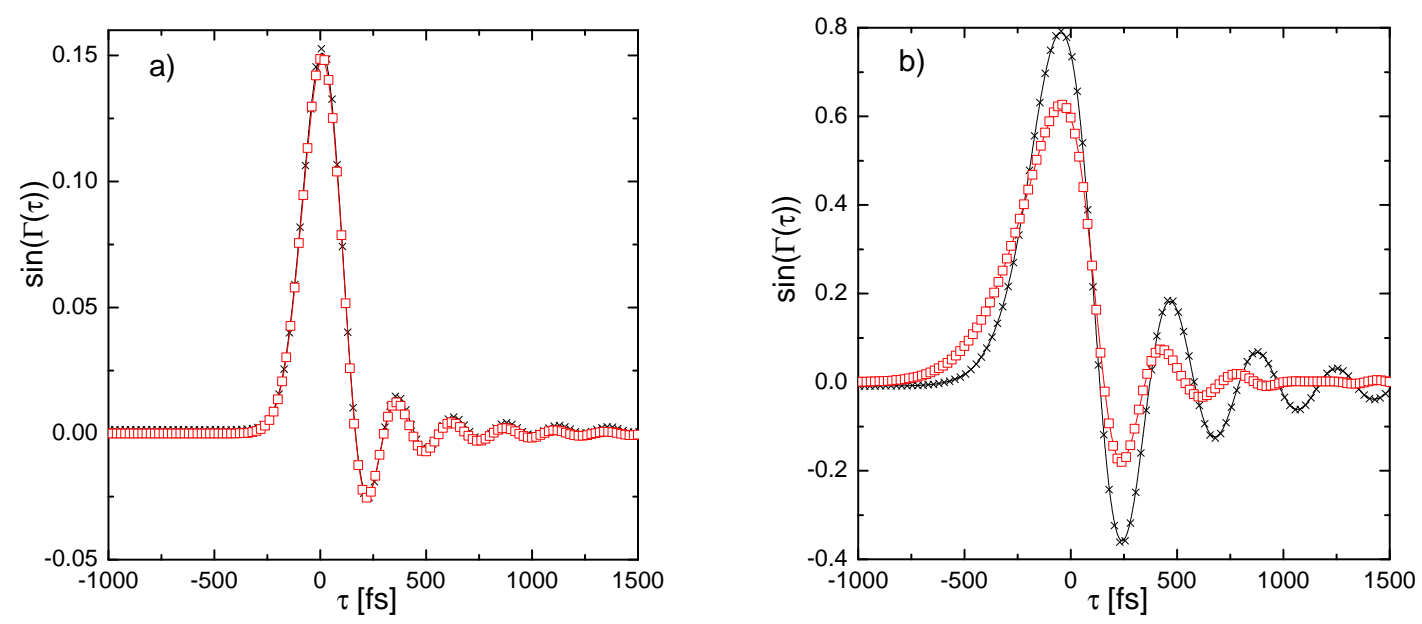

Figure 28: The balanced detector signal of ZnTe computed with the pulse propagation method (squares), taking into account the laser pulse broadening, in comparison with the response function method (crosses). Bunch charge $Q=0.2 \mathrm{nC}$, rms bunch length $\sigma_{z}=20 \mu \mathrm{m}$. a) Crystal thickness of $100 \mu \mathrm{m}$. b) Crystal thickness of $800 \mu \mathrm{m}$.

\footnotetext{
${ }^{6}$ When the number of slices tends to infinity, the sum in Eq. (36) goes over into an integral. Then the pulse propagation method is mathematically equivalent to the response function method. The laser pulse broadening can also be incorporated in the response function method.
} 


\section{References}

[1] Q. Wu and X.-C. Zhang. Free-space electro-optic sampling of terahertz beams. Appl. Phys. Lett., 67:3523, 1995.

[2] X. Yan et al. Subpicosecond electro-optic measurement of relativistic electron pulses. Phys. Rev. Lett., 85:3404, 2000.

[3] I. Wilke et al. Single-shot electron-beam bunch length measurements. Phys. Rev. Lett., 88:124801-1, 2002.

[4] A. Winter et al. Bunch length measurements at the SLS linac using electro-optical techniques. In Proceedings of the 9th European Particle Accelerator Conference (EPAC2004), Lucerne, Switzerland, 2004.

[5] B. Steffen et al. Bunch length measurements at the SLS linac using electro optical sampling. In Proceedings of the 26th International Free-Electron Laser Conference and the 11th FEL User-Workshop, FEL2004, Trieste, Italy, 2004.

[6] G. Berden et al. Electro-optic technique with improved time resolution for realtime, nondestructive, single-shot measurements of femtosecond electron bunch profiles. Phys. Rev. Lett., 93:114802, 2004.

[7] M. Brunken et al. Electro-optic sampling at the TESLA test accelerator: experimental setup and first results. TESLA Report 2003-11, 2003.

[8] D. T. Marple. Refractive index of ZnSe, ZnTe and CdTe. J. Appl. Phys., 35:539, 1964.

[9] L. Ward. Zinc Selenide (ZnSe) Zinc Telluride (ZnTe). Handbook of Optical Constants of Solids II. Harcourt Brace Jovanovich, 1991.

[10] A. N. Pikhtin et al. Dispersion of the refractive index of light and permittivity of gallium phosphide. Sov. Phys. Semicond., 10:1224, 1976.

[11] K. Strössner, S. Ves, and M. Cardona. Refractive index of GaP and its pressure dependence. Phys. Rev. B, 32:6614, 1985.

[12] D. E. Aspnes and A. A. Studna. Dielectric functions and optical parameters of Si, Ge, GaP, GaAs, GaSb, InP, InAs, and InSb from 1.5 to 6.0 eV . Phys. Rev. B, 27:985, 1983.

[13] A. S. Barker Jr. Transverse and longitudinal optic mode study in $\mathrm{MgF}_{2}$ and $\mathrm{ZnF}_{2}$. Phys. Rev., 136:A1290, 1964.

[14] G. Gallot et al. Measurements of the THz absorption and dispersion of ZnTe and their relevance to the electro-optic detection of THz radiation. Appl. Phys. Lett., 74, 1999 .

[15] T. Hattori et al. Indeces of refraction of ZnS, ZnSe, ZnSe, CdS and CdTe in the far infrared. Opt. Comm., 7:229, 1973. 
[16] M. Schall et al. Fundamental and second-order phonon processes in CdTe and ZnTe. Phys. Rev. B, 64:094301, 2001.

[17] D. A. Kleinman and W. G. Spitzer. Infrared lattice absorption of GaP. Phys. Rev., 118:110, 1960.

[18] Q. Wu and X.-C. Zhang. 7 terahertz broadband GaP electro-optic sensor. Appl. Phys. Lett., 70:14, 1997.

[19] W. L. Faust and C. H. Henry. Mixing of visible and near-resonance infrared light in GaP. Phys. Rev. Lett., 25:1265, 1966.

[20] A. Leitenstorfer et al. Detectors and sources for ultrabroadband electro-optic sampling: Experiment and theory. Appl. Phys. Lett., 74:1516, 1999.

[21] I. P. Kaminow. Measurements of the electrooptic effect in CdS, ZnTe, and GaAs at 10.6 microns. IEEE J. Quantum Electron., 4:23, 1968.

[22] K. Tada and M. Aoki. Linear electrooptic properties of ZnTe at 10.6 microns. Jap. J. Appl. Phys., 10:998, 1971.

[23] T. R. Sliker and J. M. Jost. Linear electro-optic effect and refractive indices of cubic ZnTe. J. Opt. Soc Am., 56:130, 1966.

[24] D. Berlincourt, H. Jaffe, and L. R. Shiozawa. Electroelastic properties of the sulfides, selenides, and tellurides of zinc and cadmium. Phys. Rev., 129:1009, 1963.

[25] D. F. Nelson and E. H. Turner. Electro-optic and piezoelectric coefficients and refractive index of gallium phosphide. J. Appl. Phys., 39:3337, 1968.

[26] D. Meschede. Optics, Light and Lasers. WILEY-VCH-Verlag, Weinheim, 2004.

[27] R. Li. Sensitivity of the CSR self-interaction to the local longitudinal charge concentration of an electron bunch. Nucl. Instr. and. Meth. A, 475:498, 2001.

[28] G. Geloni et al. Application of constrained deconvolution technique for reconstruction of electron bunch profile with strongly non-gaussian shape. Nucl. Instr. and. Meth. A, 528:330, 2004. 